\title{
PREDICATIVITY THROUGH TRANSFINITE REFLECTION
}

\author{
ANDRÉS CORDÓN-FRANCO, DAVID FERNÁNDEZ-DUQUE, JOOST J. JOOSTEN, \\ AND FRANCISCO FÉLIX LARA-MARTÍN
}

\footnotetext{
Abstract. Let $T$ be a second-order arithmetical theory, $\Lambda$ a well-order, $\lambda<\Lambda$ and $X \subseteq \mathbb{N}$. We use $[\lambda \mid X]^{\Lambda} \varphi$ as a formalization of " $\varphi$ is provable from $T$ and an oracle for the set $X$, using $\omega$-rules of nesting

$$
\begin{gathered}
T \\
\text { depth at most } \lambda " \text {. }
\end{gathered}
$$

For a set of formulas $\Gamma$, define predicative oracle reflection for T over $\Gamma\left(\operatorname{Pred}-0-\operatorname{RFN}_{\Gamma}(T)\right)$ to be the schema that asserts that, if $X \subseteq \mathbb{N}, \Lambda$ is a well-order and $\varphi \in \Gamma$, then

$$
\forall \lambda<\Lambda\left([\lambda \mid X]_{T}^{\Lambda} \varphi \rightarrow \varphi\right) .
$$

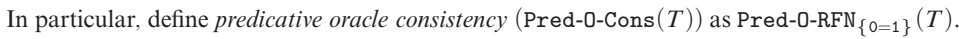

Our main result is as follows. Let $\mathrm{ATR}_{0}$ be the second-order theory of Arithmetical Transfinite Recursion, $\mathrm{RCA}_{0}^{*}$ be Weakened Recursive Comprehension and ACA be Arithmetical Comprehension with Full Induction. Then,

$$
\mathrm{ATR}_{0} \equiv \mathrm{RCA}_{0}^{*}+\operatorname{Pred-0-Cons}\left(\mathrm{RCA}_{0}^{*}\right) \equiv \mathrm{RCA}_{0}^{*}+\operatorname{Pred}_{-0-\mathrm{RFN}_{\Pi_{2}^{1}}(\mathrm{ACA})} .
$$

We may even replace $\mathrm{RCA}_{0}^{*}$ by the weaker $\mathrm{ECA}_{0}$, the second-order analogue of Elementary Arithmetic.

Thus we characterize $\mathrm{ATR}_{0}$, a theory often considered to embody Predicative Reductionism, in terms of strong reflection and consistency principles.
}

1. Introduction. Reflection over a theory $T$ roughly says that whatever is provable in $T$ is actually true. As such, reflection is natural from a methodological or philosophical point of view. Moreover, from a technical point of view it also turns out to be natural, since various well-known fragments of arithmetic can be re-cast in terms of reflection principles. In this introduction we will discuss results along these lines for first-order arithmetical theories and address the question of how this can be extended beyond first-order theories.

1.1. Reflection, consistency and fragments of first-order arithmetic. Fix a formal theory $T$. If we denote the formal provability of a formula $\varphi$ in $T$ by $\square_{T} \varphi$, we can write $\operatorname{Rfn}(T)$, called local reflection over $T$, as the scheme $\square_{T} \varphi \rightarrow \varphi$, where $\varphi$ has no free variables.

It turns out that a better-behaved notion of reflection is so-called uniform reflection where we allow for formulas, possibly with parameters. This scheme, denoted $\operatorname{RFN}(T)$, is given by $\forall x\left(\square_{T} \varphi(\dot{x}) \rightarrow \varphi(x)\right)$, where $\varphi$ is any formula and $\dot{x}$ means that we must replace $x$ by a name for $x$. 
Let us denote the consistency of $T+\varphi$ by $\diamond_{T} \varphi$, which is equivalent to $\neg \square_{T} \neg \varphi$. By Gödel's Second Incompleteness Theorem we know that consistent computably enumerable (c.e.) theories $T$ do not, in general, prove their own reflection principles; if we define $\perp$ by $0=1$, we know that $T \nvdash \square_{T} \perp \rightarrow \perp$, since the latter is provably equivalent to the consistency of $T$.

Thus, adding reflection to a consistent base theory will yield a proper extension of it. This is philosophically appealing, since one can sustain that it is natural to accept reflection over $T$ once one has accepted $T$. An early result by Kreisel and Lévy [23] shows that reflection principles are also natural from a technical point of view in that adding them yields natural extensions. Below, PA denotes the well-known firstorder theory Peano Arithmetic and PRA refers to Primitive Recursive Arithmetic, which is often considered to embody Hilbert's concept of finitist mathematics [29].

Theorem 1.1 (Kreisel, Lévy (1968)). PRA + RFN(PRA) $\equiv$ PA.

This relation between reflection and a system of arithmetic can be extended to fragments of Peano arithmetic like the theories $\mathrm{I} \Sigma_{n}$. The theory $\mathrm{I} \Sigma_{n}$ is Peano arithmetic with induction restricted to $\Sigma_{n}$-formulas. Given a class of formulas $\Gamma$, we may consider restricted reflection principles, denoted $\operatorname{RFN}_{\Gamma}(T)$, when we only consider instances of $\operatorname{RFN}(T)$ with $\varphi(x) \in \Gamma$.

Leivant [24] proved that there is a sharp correspondence between the principles I $\Sigma_{n}$ for $n \geq 2$ and restricted reflection principles over PRA. Beklemishev [2] extended Leviant's result to the case $n=1$ by lowering the base theory from PRA to a somewhat weaker theory EA called Kalmár Elementary Arithmetic.

THEOREM 1.2. Given $n \geq 1$, EA $+\operatorname{RFN}_{\Sigma_{n+1}}(\mathrm{EA}) \equiv \mathrm{I} \Sigma_{n}$.

Moreover, for various notions of provability one can often relate reflection to consistency statements. This relation is normally proved in the way presented in Lemma 1.4 below, where we need to require some minimal properties of the particular provability predicate, leading to the notion of normal provability predicate.

DEFINITION 1.3. We will call a predicate a normal provability predicate if it is

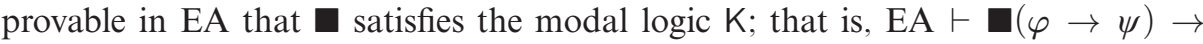
$(\boldsymbol{\square} \rightarrow \mathbf{\square})$, and EA $\vdash \varphi$ implies that EA $\vdash \mathbf{\square}$.

If $U$ is any theory and $\Gamma$ a set of formulas, we say that $\mathbf{\square}$ is provably $\Gamma$-complete in $U$ if $U \vdash \gamma \rightarrow \boldsymbol{\square} \gamma$ for any $\gamma \in \Gamma$.

Below, we will use the notation $\neg \Gamma=\{\neg \gamma: \gamma \in \Gamma\}$.

LEMMA 1.4. Let $U$ be a theory extending EA and let $\mathbf{\square}$ be a normal provability predicate with dual consistency predicate $\downarrow$. If $\Gamma$ contains (a provable equivalence of $)$ $\perp$ and $\mathbf{\square}$ is provably $\neg \Gamma$-complete in $U$, then $\Gamma$-reflection for $\mathbf{\square}$ is equivalent over $U$ to $\checkmark$ T. That is, $U+\{\boldsymbol{\square} \rightarrow \gamma: \gamma \in \Gamma\} \equiv U+\downarrow \top$.

Proof. The $\vdash$ direction follows directly from the assumption that $\perp \in \Gamma$. The other direction follows from $\neg \Gamma$-completeness: suppose that $\boldsymbol{\sigma}$ and $\top$ and suppose for a contradiction that $\neg \gamma$; by completeness we have $\boldsymbol{\nabla}$, whence by $\boldsymbol{\nabla} \gamma$ and by normality we get $\boldsymbol{\square}$, which contradicts $\checkmark T$.

Stronger notions of provability can be related to stronger notions of consistency. For this purpose it is very useful to consider the provability predicates $[n]_{T}$ for $n \in \omega$ and c.e. theories $T$ where $[n]_{T}$ is a natural first-order formalization of "provable 
from the axioms of $T$ together with some true $\Pi_{n}$ sentence". More precisely, let True $_{\Pi_{n}}$ be the standard partial truth-predicate for $\Pi_{n}$ formulas, which is itself of complexity $\Pi_{n}$ (see [18] for information about partial truth definitions within EA). Then, we define

$$
[n]_{T} \varphi \leftrightarrow \exists \pi\left(\operatorname{True}_{\Pi_{n}}(\pi) \wedge \square_{T}(\pi \rightarrow \varphi)\right) .
$$

For each c.e. theory $T$ extending EA and $n \in \omega$, the predicate $[n]_{T}$ is normal and provably $\Pi_{n}$-complete in EA (for proofs see [5, Propositions 2.10 and 2.11]). Hence, together with Lemma 1.4, we get

$$
T+\langle n\rangle_{T} \top \equiv T+\left\{\forall x\left([n]_{T} \sigma(\dot{x}) \rightarrow \sigma(x)\right): \sigma \in \Sigma_{n}\right\} .
$$

We can easily prove that the reflection principle from (1) is equivalent over $T$ to the more standard notion of reflection $\operatorname{RFN}_{\Sigma_{n}}(T)$. Thus, together with Theorem 1.2, we see the intimate relation between reflection, consistency statements and fragments of first-order arithmetic.

THeOREM 1.5. Given $n \geq 1$, EA $+\langle n+1\rangle_{\mathrm{EA}} \top \equiv \mathrm{EA}+\operatorname{RFN}_{\Sigma_{n+1}}(\mathrm{EA}) \equiv \mathrm{I} \Sigma_{n}$.

1.2. Ordinal analysis beyond first-order. Ignatiev showed in [20] that for a large variety of theories $T$, the joint behavior of the provability predicates $[n]_{T}$ for $n \in \omega$ can be described and fully understood via a relatively simple and well-behaved modal logic called GLP, first studied by Japaridze in [21]. Theorem 1.5 is a clear witness of the expressiveness of this modal logic; as a matter of fact, Beklemishev has shown in [3] that the computation of a proof-theoretical $\Pi_{1}^{0}$ ordinal for PA along the lines of Schmerl's work [25] on transfinitely adding consistency to a weak base theory can be realized largely within GLP.

The ordinals involved in the analysis actually are naturally represented within the logic GLP by so-called worms, which are iterated consistency statements of the form $\left\langle n_{1}\right\rangle \ldots\left\langle n_{m}\right\rangle \top$. These iterated consistency statements are called worms in analogy to hydra's in the hydra battle since they give rise to a similar worm battle with corresponding unprovable true combinatorial principles and related term-rewriting systems $([6,9])$.

However, worms in GLP correspond to ordinals below $\varepsilon_{0}$, so GLP would certainly not suffice for the analysis of theories which are substantially stronger than PA. First steps to extend the relation between ordinals and modal-logical terms beyond $\varepsilon_{0}$ were made in $[4,8,14,15,17]$ by studying logics $G L P_{\Lambda}$ that contain for each ordinal $\alpha<\Lambda$ a modality [ $\alpha$ ]. One should go beyond first-order theories in order to interpret $G L P_{\Lambda}$, and in [16] two of the authors provide an interpretation of GLP $\Lambda$ within second-order arithmetic by reading $[\alpha]_{T} \phi$ as $\phi$ is derivable in $T$ using an $\omega$-rule of depth at most $\alpha$.

Our present goal is to provide analogues of Theorem 1.5 for fragments of secondorder arithmetic, by using more powerful reflection principles. These principles will involve provability using transfinite iterations of the $\omega$-rule and require reasoning about arbitrary sets in the guise of free set parameters, which poses a challenge in a proof-theoretic setting. Every natural number can be represented by a closed term; however, in any countable language it is of course not the case that each set can be denoted by a syntactical name.

In [1] this problem was addressed by resorting to a richer language with sufficiently many names around. Our way to surpass this complication is by introducing and 
studying what we call oracle provability which allows us to reason in a formalized setting about externally defined sets. In particular, we will introduce a new firstorder predicate $\mathcal{O}$ which we shall call the oracle predicate and which will be used to reason formally about arbitrary sets.

Once a workable relation between reflection principles and fragments of second order arithmetic has been established, this relation will constitute a significant step forward in the $\Pi_{1}^{0}$ ordinal analysis and semifinitary consistency proofs of secondorder theories as anticipated in [22]. A next ingredient towards such an ordinal analysis will be to study one of the forms of the so-called reduction property (see e.g., [7,22]) which expresses the amount of conservativity between various fragments of second order arithmetic based on the consistency statements that are introduced in this paper.

1.3. Overview of the paper. In Section 2 we will settle our notation and nomenclature and fix the formal language that we will work in. One new ingredient is the first-order oracle symbol $\mathcal{O}$ which will, in a way, provide a name for any arbitrary set. We define formalized oracle provability in this new language and mention some basic properties.

In Section 3 we will define the fragments of second-order arithmetic which are relevant for this paper, and in Section 4 we formalize within these fragments the notion $[\lambda \mid X]_{T}^{\Lambda}$ of provability in $T$ using an oracle for $X$ and at most $\lambda<\Lambda$ iterations of the $\omega$-rule. Next, in Section 5 we define and prove the basic properties of the notions of reflection and consistency based on the provability predicates $[\lambda \mid X]_{T}^{\Lambda}$.

In Section 6 we define the notions of predicative oracle reflection and consistency. Basically, predicative oracle reflection/consistency is the statement that "if $\Lambda$ is a well-order, then reflection/consistency holds for iterations of the $\omega$-rule along $\Lambda$ with an oracle for $X$ ". In the section we show that predicative oracle consistency proves $\mathrm{ATR}_{0}$, the second-order system of arithmetical transfinite recursion. Finally, in Section 7 we prove the converse implication so that we end up with a characterization of $\mathrm{ATR}_{0}$ in terms of predicative oracle reflection and consistency.

It follows from Theorem 1.5 that PA $\equiv \mathrm{EA}+\left\{\langle n\rangle_{\mathrm{EA}} \top: n<\omega\right\}$. Our results show that $\mathrm{ATR}_{0}$ can similarly be reduced to sufficiently strong consistency or reflection principles. Using slightly suggestive notation, one can paraphrase our result as

$\mathrm{ATR}_{0} \equiv \mathrm{ECA}_{0}+$ " $\Lambda$-OracleCons $\left(\mathrm{ECA}_{0}\right)$ holds for every well-order $\Lambda$ ",

where $\mathrm{ECA}_{0}$ is the second-order pendant of $\mathrm{EA}$, and $\Lambda$-OracleCons $\left(\mathrm{ECA}_{0}\right)$ denotes oracle consistency for $\mathrm{ECA}_{0}$ for iterations of the $\omega$-rule along $\Lambda$.

2. Second-order theories with oracles. In this section we fix syntactical notations for the remainder of this paper. Moreover, we will formalize a notion of provability where we use an oracle symbol which provides a name for an arbitrary set much like one has numerals to name natural numbers.

2.1. Conventions of syntax. We will work in a language $\mathcal{L}_{2}$ of second-order arithmetic, with primitive symbols $0,1,+, \times, x^{y},<,=, \in$, representing the standard constants, operations and relations on the natural numbers; that is, $\mathcal{L}_{2}$ extends the usual language of second-order arithmetic with a primitive symbol for exponentiation. 
Moreover, we will be interested in an extension of second-order arithmetic, $\mathcal{L}_{2}(\mathcal{O})$, which contains a designated unary predicate $\mathcal{O}(\cdot)$, used to represent a set "oracle", as specified below.

We fix some set of numerals which are terms so that each natural number $n$ is denoted by exactly one numeral, written as $\bar{n}$. Moreover, we fix some Gödel numbering, mapping a formula $\psi \in \mathcal{L}_{2}(\mathcal{O})$ to its corresponding Gödel number $\ulcorner\psi\urcorner$, and similarly for terms and sequents of formulas (used to represent derivations). We will assume that the Gödel numbering is simple in that simple syntactical operations like concatenation and substitution correspond to elementary functions on the respective Gödel numbers. Further, we will assume that the Gödel numbering is natural in that the Gödel number of a strict substring is numerically smaller than the Gödel number of the entire string and, moreover, that $n<\ulcorner\bar{n}\urcorner$. Since we will be working mainly inside theories of arithmetic, we will often identify $\psi$ with $\ulcorner\psi\urcorner$, or even with $\overline{\ulcorner\psi\urcorner}$, for that matter.

$\Delta_{0}^{0}$ denotes the set of all $\mathcal{L}_{2}$ formulas, possibly with set parameters, but without the occurrence of $\mathcal{O}$, and where no second-order quantifiers appear and all firstorder quantifiers are bounded; that is, of the form $\forall x<y \varphi$ or $\exists x<y \varphi$. We simultaneously define $\boldsymbol{\Sigma}_{0}^{0}=\boldsymbol{\Pi}_{0}^{0}=\boldsymbol{\Delta}_{0}^{0}$ and recursively define $\boldsymbol{\Sigma}_{n+1}^{0}$ to be the set of all formulas of the form $\exists x_{0} \cdots \exists x_{m} \varphi$ with $\varphi \in \boldsymbol{\Pi}_{n}^{0}$, and similarly $\boldsymbol{\Pi}_{n+1}^{0}$ to be the set of all formulas of the form $\forall x_{0} \cdots \forall x_{m} \varphi$ with $\varphi \in \boldsymbol{\Sigma}_{n}^{0}$. We denote by $\boldsymbol{\Pi}_{\omega}^{0}$ the union of all $\boldsymbol{\Pi}_{n}^{0}$; these are the arithmetical formulas.

The classes $\boldsymbol{\Sigma}_{n}^{1}, \boldsymbol{\Pi}_{n}^{1}, \boldsymbol{\Pi}_{\omega}^{1}$ are defined analogously but using second-order quantifiers and setting $\boldsymbol{\Sigma}_{0}^{1}=\boldsymbol{\Pi}_{0}^{1}=\boldsymbol{\Delta}_{0}^{1}=\boldsymbol{\Pi}_{\omega}^{0}$. It is well-known that every second-order formula is equivalent to another in one of the above forms. We use a lightface font for the analogous classes where no set-variables appear free: $\Delta_{0}^{m}, \Pi_{n}^{m}, \Sigma_{n}^{m}$. The set of free variables of a formula $\varphi$ will be denoted by $\operatorname{FV}(\varphi)$.

We also use $\Delta_{0}^{0}\left(X_{1}, \ldots, X_{k}\right)$ to denote the class of those $\Delta_{0}^{0}$ formulas whose free second order variables are among $X_{1}, \ldots, X_{k}$, and analogously for the classes $\Pi_{n}^{m}\left(X_{1}, \ldots, X_{k}\right), \Sigma_{n}^{m}\left(X_{1}, \ldots, X_{k}\right)$, etc.

We will say that a theory $T$ is elementarily presented if a $\Delta_{0}^{0}$ formula $\operatorname{Axiom}_{T}(x)$ is specified that is true if and only if $x$ codes a (non-logical) axiom of $T$. Then, there is a $\Delta_{0}^{0}$ formula $\operatorname{Proof}_{T}(x, y)$ which holds if and only if $x$ codes a derivation in $T$ of a formula coded by $y$. Note that by Craig's trick, any c.e. theory has an equivalent elementary presentation. We will write $\square_{T} \varphi$ as shorthand for $\exists p \operatorname{Proof}_{T}(p, \varphi)$.

We will also use the following pseudo-terms to simplify notation, where an expression $\varphi(t(\vec{x}))$ should be seen as a shorthand for $\exists y<s(\vec{x})(\psi(\vec{x}, y) \wedge \varphi(y))$, with $\psi$ a $\Delta_{0}^{0}$ formula defining the graph of the intended interpretation of $t$ and $s$ a standard term bounding the values of $t(\vec{x})$ :

1. A term $\langle x, y\rangle$ which returns a code of the ordered pair formed by $x$ and $y$ and projection terms so that $(\langle x, y\rangle)_{0}=x$ and $(\langle x, y\rangle)_{1}=y$. We may also write $t_{i}$ instead of $(t)_{i}$ when this improves legibility.

2. A term $x[y / z]$ which, when $x$ codes a formula $\varphi(v), y$ a variable $v$ and $z$ a term $t$, returns the code of $\varphi(t)$. Otherwise, its value is unspecified, or for example it could be the default $\ulcorner\perp\urcorner$.

3. A term $x \rightarrow y$ which, when $x, y$ are codes for $\varphi, \psi$, returns a code of $\varphi \rightarrow \psi$, and similarly for other connectives and quantifiers. 
4. A term $\bar{x}$ mapping a number to the code of its numeral.

5. For every formula $\varphi$ and variables $x_{0}, \ldots, x_{m}$, a term $\varphi\left(\dot{x}_{0}, \ldots, \dot{x}_{m}\right)$ which, given numbers $n_{0}, \ldots, n_{m}$, returns the code of the outcome of $\varphi[\vec{x} / \overrightarrow{\vec{n}}]$, i.e., the code of $\varphi\left(\bar{n}_{0}, \ldots, \bar{n}_{m}\right)$.

The only purpose of using these pseudo-terms is to shorten complex formulas for the sake of legibility. We write $\square_{T} \varphi\left(\dot{x}_{0}, \ldots, \dot{x}_{n}\right)$ as shorthand for $\exists \psi(\psi=$ $\left.\varphi\left(\dot{x}_{0}, \ldots, \dot{x}_{n}\right) \wedge \square_{T} \psi\right)$ and adhere to the same convention when using other notions of provability. Note that $\square_{T} \varphi\left(\dot{x}_{0}, \ldots, \dot{x}_{n}\right)$ is a formula, not a term.

2.2. Oracle provability. The oracle $\mathcal{O}$ will be used to add information about any set of numbers to our theory $T$. To be precise, given a set $A \subseteq \mathbb{N}$, define $T \mid A$ to be the theory

$$
T+\{\mathcal{O}(\bar{n}): n \in A\}+\{\neg \mathcal{O}(\bar{n}): n \notin A\}+\{\exists Y \forall x(x \in Y \leftrightarrow \mathcal{O}(x))\} .
$$

It should be clear that if $T$ is elementary presented then $T \mid X$ has a $\Delta_{0}^{0}(X)$ axiomatization (i.e., the only set variable that may appear is $X$ ) and provability in $T \mid X$ is $\Sigma_{1}^{0}(X)$. To be more precise, there exist formulas $\operatorname{Axiom}_{T \mid X}(x) \in \Delta_{0}^{0}(X)$, as well as $\operatorname{Proof}_{T \mid X}(x, y) \in \Delta_{0}^{0}(X)$, and $\square_{T \mid X}(x) \in \Sigma_{1}^{0}(X)$ so that $\varphi$ is an axiom of $T \mid A$ if and only if $\mathbb{N}=\operatorname{Axiom}_{T \mid A}(\ulcorner\varphi\urcorner)$, the sequence $\pi$ is a proof of $\varphi$ in the theory $T \mid A$ if and only if $\mathbb{N}=\operatorname{Proof}_{T \mid A}(\ulcorner\pi\urcorner,\ulcorner\varphi\urcorner)$, and $T \mid A \vdash \varphi$ if and only if $\mathbb{N} \models \square_{T \mid A}(\ulcorner\varphi\urcorner)$. For example, we can define

$$
\begin{aligned}
& \operatorname{Axiom}_{T \mid X}(\varphi):=\operatorname{Axiom}_{T}(\varphi) \vee \exists x<\varphi(\varphi=\ulcorner\mathcal{O}(\bar{x})\urcorner \wedge x \in X) \\
& \vee \exists x<\varphi(\varphi=\ulcorner\neg \mathcal{O}(\bar{x})\urcorner \wedge x \notin X) \vee \varphi=\ulcorner\exists Y \forall x(x \in Y \leftrightarrow \mathcal{O}(x))\urcorner,
\end{aligned}
$$

so that $\operatorname{Proof}_{T \mid X}(x, \varphi)$ will just become as $\operatorname{Proof}_{T}(x, \varphi)$ where every occurrence of $\operatorname{Axiom}_{T}(\pi)$ for some $\pi$ is replaced by $\operatorname{Axiom}_{T \mid X}(\pi)$. As before, we define $\square_{T \mid X}(\varphi)$ by $\exists x \operatorname{Proof}_{T \mid X}(x, \varphi)$. For readability, when working in $T \mid A$ we will write $x \in \bar{A}$ instead of $\mathcal{O}(x)$, provided this does not lead to confusion.

As is standard, one may use a single set $\left\{\langle i, x\rangle: x \in A_{i}\right\}$ to represent sets $A_{1}, \ldots, A_{n}$, and as such we will freely use our oracle to interpret tuples of sets. If working in $T \mid A_{1}, \ldots, A_{n}$, we will write $x \in \bar{A}_{i}$ instead of $\mathcal{O}(\langle i, x\rangle)$.

However, a word of warning is due here. The bar notation we have introduced is informal and will not be used in ambiguous expressions like $\square_{T \mid A . A}(x \in \bar{A})$ or $\square_{T \mid X}\left(x \in X \rightarrow \square_{T \mid X} \dot{x} \in \bar{X}\right)$. Fortunately, for the purpose of this paper these ambiguities are not salient, and the notation will be useful in making expressions easier to understand.

Finally, we mention that we would like to be able to infer $\square_{T \mid X} \exists Y \varphi(Y)$ from the formula $\square_{T \mid X} \varphi(\bar{X})$. This is why in $T \mid X$ we have included the axiom $\exists Y \forall x(x \in Y \leftrightarrow \mathcal{O}(x))$.

3. Fragments of second-order arithmetic. Important fragments of second-order arithmetic are typically characterized by their set-existence axioms. In this section we shall introduce the fragments which are relevant for this paper.

3.1. Comprehension and induction. It is important to keep track of the secondorder principles that are used; below we describe the most basic ones. The induction and comprehension axioms are the universal closures of the following formulas. 
$\Gamma$-CA $\quad \exists X \forall x(x \in X \leftrightarrow \varphi(x)) \quad$ where $\varphi \in \Gamma$ and $X$ is not free in $\varphi$;

$\Delta_{1}^{0}$-CA $\forall x(\varphi(x) \leftrightarrow \psi(x)) \rightarrow \exists X \forall x(x \in X \leftrightarrow \varphi(x))$ where $\varphi \in \Sigma_{1}^{0}, \psi \in \mathbf{\Pi}_{1}^{0}$,

and $X$ is not free in $\varphi$;

I $\quad \varphi(0) \wedge \forall x(\varphi(x) \rightarrow \varphi(x+1)) \rightarrow \forall x \varphi(x) \quad$ where $\varphi \in \Gamma$;

Ind $0 \in X \wedge \forall x(x \in X \rightarrow x+1 \in X) \rightarrow \forall x(x \in X)$.

We assume all theories extend two-sorted classical first-order logic, so that they include modus ponens, generalization, etc., as well as Robinson arithmetic Q, i.e., Peano arithmetic without induction. By $\mathrm{Q}^{+}$we denote $\mathrm{Q}$ together with the defining axioms for $x^{y}$.

In the list below, recall that we have included exponentiation in our language, which is essential for the theories $\mathrm{ECA}_{0}$ and $\mathrm{RCA}_{0}^{*}$.

$$
\begin{array}{llll}
\mathrm{ECA}_{0}: & \mathrm{Q}^{+}+\text {Ind }+\Delta_{0}^{0}-\mathrm{CA} ; & \mathrm{ACA}_{0}: & \mathrm{Q}^{+}+\mathrm{Ind}+\boldsymbol{\Pi}_{\omega}^{0}-\mathrm{CA} \\
\mathrm{RCA}_{0}^{*}: & \mathrm{Q}^{+}+\mathrm{Ind}+\Delta_{1}^{0}-\mathrm{CA} ; & \mathrm{ACA}: & \mathrm{Q}^{+}+\mathrm{I} \boldsymbol{\Pi}_{\omega}^{1}+\boldsymbol{\Pi}_{\omega}^{0}-\mathrm{CA} . \\
\mathrm{RCA}_{0}: & \mathrm{Q}^{+}+\mathrm{I} \boldsymbol{\Sigma}_{1}^{0}+\Delta_{1}^{0}-\mathrm{CA} ; & &
\end{array}
$$

We mention these from weakest to strongest, but note that $\mathrm{ACA}_{0}$ is still a mediumstrength system of second-order arithmetic, being arithmetically conservative over $\mathrm{PA}$. The system $\mathrm{ECA}_{0}$ stands for Elementary Comprehension Axiom and was introduced in [16] as the second-order equivalent of Elementary Arithmetic, EA (we refer the reader to [5] for an axiomatization of EA). In order to relate $\mathrm{ECA}_{0}$ to the more classical systems we need to mention $\Sigma_{1}^{0}$-bounding. The principle of $\Sigma_{1}^{0}$-bounding is given by

$$
\forall x<a \exists z \varphi(x, y, z) \rightarrow \exists b \forall x<a \exists z<b \varphi(x, y, z)
$$

with $\varphi \in \Sigma_{1}^{0}$, and is also referred to as $\Sigma_{1}^{0}$-collection.

LEMMA 3.1. The first-order part of $\mathrm{ECA}_{0}$ is $\mathrm{EA}$, and the first-order part of $\mathrm{RCA}_{0}^{*}$ is EA plus $\Sigma_{1}^{0}$-bounding. Moreover, $\mathrm{RCA}_{0}^{*}$ is $\Pi_{2}^{0}$-conservative over $\mathrm{ECA}_{0}$, and $\Sigma_{2}^{0}$ sentences are already not conserved.

ProOF SKETCH. In [28] the above-mentioned characterization of the first-order part of $\mathrm{RCA}_{0}^{*}$ is proven. If $\mathfrak{M}$ is a model of EA, then it is easy to check that $\langle\mathfrak{M}, \mathcal{S}\rangle$ is a model of $\mathrm{ECA}_{0}$, where $\mathcal{S}$ is the set of all $\Delta_{0}^{0}$-definable subsets of $\mathfrak{M}$. This proves that the first-order part of $\mathrm{ECA}_{0}$ is just EA.

It is well-known (see e.g., [18], Section 1(f), Chapter IV) that EA plus $\Sigma_{1}^{0}$-bounding is $\Pi_{2}^{0}$ conservative over EA. Moreover, the least number principle for parameter-free $\Delta_{1}^{0}$ predicates is known to be $\Sigma_{2}^{0}$-axiomatizable, provable from $\Sigma_{1}^{0}$-bounding over EA, but not provable in plain EA (see e.g., [11]). This establishes that $\Sigma_{2}^{0}$ conservativity fails.

With the dot notation from above we can express an important feature of formalized provability called provable $\Sigma_{1}$-completeness which says that true $\Sigma_{1}^{0}$ formulas are actually provable.

Lemma 3.2 (Provable $\Sigma_{1}$-completeness). Let $U$ be a theory in the language of arithmetic that extends $\mathrm{ECA}_{0}$. Further, let $T$ be an elementary presented theory extending Robinson arithmetic. For any $\Sigma_{1}^{0}$ formula $\sigma\left(x_{1}, \ldots, x_{n}\right)$, we have that

$$
U \vdash \sigma\left(x_{1}, \ldots, x_{n}\right) \rightarrow \square_{T \mid X} \sigma\left(\dot{x}_{1}, \ldots, \dot{x}_{n}\right) .
$$

A proof of EA-provable $\Sigma_{1}$ completeness for regular provability $\square_{T}$ can be found, e.g., in [18] or in [10] where in the latter reference we only need to observe that all 
arguments only require the totality of exponentiation and very little induction. Since $T \mid X$ is provably an extension of $T$ we get Lemma 3.2. One key ingredient in the proof is that bounded quantifiers may be effectively replaced by finite conjunctions and disjunctions; the latter remains true in our second-order context, so we mention it explicitly in the form that we will use it.

Lemma 3.3. Let $T$ be any theory extending $\mathrm{Q}$ and $\varphi(x) \in \mathbf{\Pi}_{\omega}^{0}$. Then, it is provable in $\mathrm{ECA}_{0}$ that $\forall v \square_{T}\left(\forall u<\dot{v} \varphi(u) \leftrightarrow \bigwedge_{u<\dot{v}} \varphi(\dot{u})\right)$.

Proof. See item (5) in the proof of Lemma III.3.14 of [18]. Note that the addition of second-order or unbounded quantifiers does not affect the proof.

3.2. Transfinite induction and transfinite recursion. Another principle that will be relevant in this work is transfinite recursion, but this is a bit more elaborate to describe. Let us recall that $\mathcal{L}_{2}$ contains only monadic set-variables. Binary relations and functions are represented by coding pairs of numbers. We can also encode countable ordinals as well-orders on $\mathbb{N}$, which may be represented using a set $\Lambda$ coding a pair $\left\langle|\Lambda|,<_{\Lambda}\right\rangle$. As is standard, we write $x<_{\Lambda} y$ instead of $\langle x, y\rangle \in<_{\Lambda}$. Let linear $(\Lambda)$ be a formula naturally asserting that $\Lambda$ is a linearly ordered set, and define the formula wo $(\Lambda)$ as

$$
\operatorname{linear}(\Lambda) \wedge \forall X \subseteq|\Lambda|\left(\exists x \in X \rightarrow \exists y \in X \forall z \in X\left(y \leq_{\Lambda} z\right)\right) .
$$

We will use lower-case Greek letters for elements of $|\Lambda|$ and boldface natural numbers to denote finite ordinals, so that $\boldsymbol{n}$ is $\{0, \ldots, n-1\}$ with the usual ordering. Similarly, a boldface $\omega$ is the standard ordering on the naturals.

When it is clear from the context that we are working within $\Lambda$, we will often omit explicit mention of it and we may write $\xi<\zeta$ instead of $\xi<_{\Lambda} \zeta$; similarly we may write $\xi<\Lambda$ instead of $\xi \in|\Lambda|$. We define $x=0_{\Lambda}$ to be a formula stating that $x$ is the least element with respect to $<_{\Lambda}$, and $\operatorname{Lim}_{\Lambda}(x)$ to be a formula expressing that $x$ has limit order-type within $\Lambda$, that is,

$$
\operatorname{Lim}_{\Lambda}(x):=\quad x<\Lambda \wedge x \neq 0_{\Lambda} \wedge \forall y<_{\Lambda} x \exists z\left(y<_{\Lambda} z \wedge z<_{\Lambda} x\right) .
$$

Along these lines, we define $\operatorname{Lim}(\Lambda)$ to state that $\Lambda$ is a well-order with limit order-type.

In this paper we shall use some very basic results on ordinal addition provable in $\mathrm{ACA}_{0}$. The reverse mathematics of ordinal arithmetic has been developed by J. L. Hirst in a series of papers (see [19] for a survey and further references). However, Hirst defines ordinal addition, multiplication, and exponentiation through operations between well-orders (encoding ordinals). Here, instead, we are interested in the inner additive structure of limit ordinals. As a matter of fact, we will only need some basic properties of the function $(n, \alpha, m) \mapsto n \cdot \alpha+m$, for $n, m \in \mathbb{N}$, $\alpha<\Lambda$, and $\Lambda$ a limit ordinal. A word of warning is due: when we write $n \cdot \alpha+m$, the operations in this expressions are not the usual ordinal operations, but rather special operations on $\Lambda$ that we define below and which behave analogously.

The theory $\mathrm{ACA}_{0}$ proves that if $\operatorname{Lim}(\Lambda)$ holds then the usual successor function Succ : $|\Lambda| \rightarrow|\Lambda|$ exists and can be iterated to produce a function from $\mathbb{N} \times|\Lambda|$ to $|\Lambda|$ satisfying the recurrence equations

$$
\operatorname{Succ}^{0}(\alpha)=\alpha, \operatorname{Succ}^{k+1}(\alpha)=\operatorname{Succ}\left(\operatorname{Succ}^{k}(\alpha)\right)
$$


The following lemma summarizes the properties of the function $n \cdot \alpha+m$ that we will use. The proof is straightforward and we omit it.

LEMma 3.4. Within $\mathrm{ACA}_{0}$ we have that if $\operatorname{Lim}(\Lambda)$ holds, then:

1. For each $\alpha<\Lambda$ there are unique $k \in \mathbb{N}$ and $\beta<\Lambda$ such that $\beta=0 \vee \operatorname{Lim}_{\Lambda}(\beta)$ and $\alpha=\operatorname{Succ}^{k}(\beta)$.

2. There is a function $f: \mathbb{N} \times \Lambda \times \mathbb{N} \rightarrow \Lambda$ such that $f(n, \alpha, m)=\operatorname{Succ}^{n \cdot k+m}(\beta)$, where $\alpha=\operatorname{Succ}^{k}(\beta)$ and $\beta=0 \vee \operatorname{Lim}_{\Lambda}(\beta)$. (We will write $n \cdot \alpha+m$ instead of $f(n, \alpha, m)$ and simply $n \cdot \alpha$ when $m=0$.)

3. For all $\alpha, \beta<\Lambda$ and $n, m \in \mathbb{N}, \alpha \leq_{\Lambda} \beta \rightarrow n \cdot \alpha+m \leq_{\Lambda} n \cdot \beta+m$.

Our main focus will be the theory $\mathrm{ATR}_{0}$, in which new sets may be defined using transfinite recursion. Transfinite recursion is the principle that sets may be defined by iterating a formula along a well-order. To formalize this, let us consider a set $X$ whose elements are of the form $\langle\xi, x\rangle$. We shall write $x \in X_{\xi}$ for $\langle\xi, x\rangle \in X$

Given a formula $\varphi(X)$, define $\varphi\left(X_{<_{\lambda} \lambda}\right)$ to be the formula where every occurrence of $t \in X$ in $\varphi$ is replaced by $\left((t)_{0}<_{\Lambda} \lambda\right) \wedge(t \in X)$. Then define $\operatorname{TR}^{\Lambda}(\varphi, X)$ to be the formula

$$
\forall \xi<\Lambda \forall x\left(x \in X_{\xi} \leftrightarrow \varphi\left(x, X_{<_{\Lambda} \xi}\right)\right) .
$$

Finally, given a set of formulas $\Gamma$ we define the schema

$$
\operatorname{TR}-\Gamma: \quad \forall \Lambda\left(\text { wo }(\Lambda) \rightarrow \exists Y \operatorname{TR}^{\Lambda}(\varphi, Y)\right) \text { for } \varphi \in \Gamma .
$$

We can now write down the axiom schema for Arithmetical Transfinite Recursion:

$$
\mathrm{ATR}_{0}: \quad \mathrm{Q}^{+}+\text {Ind }+\mathrm{TR}-\Pi_{\omega}^{0} .
$$

The system $\mathrm{ATR}_{0}$ is important within reverse mathematics and commonly associated with Predicative Reductionism [12, 13, 26].

In various proofs we will reason by induction along a well-order. $\operatorname{By~}^{\Lambda}(\varphi)$ we denote the transfinite induction axiom for $\varphi$ along the ordering $<_{\Lambda}$ :

$$
\operatorname{TI}^{\Lambda}(\varphi):=\left(\forall \xi<\Lambda\left(\forall \zeta<_{\Lambda} \xi \varphi(\zeta) \rightarrow \varphi(\xi)\right)\right) \rightarrow \forall \xi<\Lambda \varphi(\xi) .
$$

We will write $\varphi$-CA instead of $\{\varphi\}$-CA, i.e., the instance of the comprehension axiom stating that $\{x: \varphi(x)\}$ is a set. The following easy lemma tells us that we have access to transfinite induction for formulas of the right complexity:

LEMMA 3.5. In any second-order arithmetical theory we can prove

$$
\text { wo }(\Lambda) \wedge(\neg \varphi)-\mathrm{CA} \rightarrow \operatorname{TI}^{\Lambda}(\varphi) .
$$

As an easy corollary to this lemma we see that in $\mathrm{ACA}_{0}$ we can apply transfinite induction for arithmetical formulas over any well-order, and we shall use this time and again in the remainder of this paper.

4. Nested $\omega$-rules for oracle provability. In this section, we will briefly discuss how nested $\omega$-rules for oracle provability can be formalized and prove certain basic properties of the formalization. From now on, $T$ will denote an elementarily presented theory extending $\mathrm{ECA}_{0}$. 
4.1. Formalizing nested $\omega$-rules for oracle provability. We will use $[\lambda]_{T}^{\Lambda} \varphi$ to denote our representation of " $\varphi$ is provable in $T$ using iterated applications of the $\omega$-rule of depth at most $\lambda$ (according to $<_{\Lambda}$ )". The desired recursion for such a sequence of provability operators is given by the following equivalence:

$$
[\lambda]_{T}^{\Lambda} \varphi \leftrightarrow\left(\square_{T} \varphi \vee \exists \psi \exists \xi<_{\Lambda} \lambda\left(\forall n[\xi]_{T}^{\Lambda} \psi(\dot{n}) \wedge \square_{T}(\forall x \psi(x) \rightarrow \varphi)\right)\right) .
$$

In [16], two of the authors formalize a notion $[\lambda]_{T}^{\Lambda} \varphi$ in second-order number theory so that under certain conditions it provably satisfies (2). In the current paper we introduce a minor modification, and define $[\lambda \mid X]_{T}^{\Lambda} \varphi=[\lambda]_{T \mid X} \varphi$. Although [16] does not consider theories with oracles, the construction given there readily applies in this setting. For the sake of clarity and keeping the paper self-contained, we shall sketch here how such a formalization would proceed and refer for [16] for further details.

As a first step in such a formalization, we will use a set $P$ as a provability operator for the oracle $X$. Its elements are codes of pairs $\langle\lambda, \varphi\rangle$, with $\lambda$ a code for an ordinal and $\varphi$ a code for a formula. We use $[\lambda]_{P} \varphi$ to denote $\langle\lambda, \varphi\rangle \in P$.

The idea is that we want to consider those sets $P$ of pairs $\langle\lambda, \varphi\rangle$ so that (2) holds for $T \mid X$ whenever we define $[\lambda \mid X]_{T}^{\Lambda} \varphi:=\langle\lambda, \varphi\rangle \in P$. Of course, we will use secondorder logic to impose the necessary conditions on the set $P$. Whenever $P$ satisfies (2) for $T \mid X$ we will write $\operatorname{IPC}_{T \mid X}^{\Lambda}(P)$ and say that " $P$ is an iterated oracle provability class". The following definition is a minor modification from [16].

Definition 4.1. Let $\Lambda$ be a second-order variable that will be used to denote a well-order. Define $\operatorname{Rule}_{T \mid X}^{\Lambda}(d, \xi, \lambda, \psi, \varphi, P)$ to be the formula

$$
\xi<_{\Lambda} \lambda \wedge \forall n[\xi]_{P} \psi(\dot{n}) \wedge \operatorname{Proof}_{T \mid X}(d, \forall x \psi(x) \rightarrow \varphi),
$$

$\operatorname{Proof}_{T \mid X}^{\Lambda}(c, \lambda, \varphi, P)$ to be

$$
\exists d \exists \xi \exists \psi\left(c=\langle d, \xi, \psi\rangle \wedge\left[\operatorname{Proof}_{T \mid X}(d, \varphi) \vee \operatorname{Rule}_{T \mid X}^{\Lambda}(d, \xi, \lambda, \psi, \varphi, P)\right]\right),
$$

and let $\operatorname{IPC}_{T \mid X}^{\Lambda}(P)$ be the formula

$$
\forall \lambda<\Lambda \forall \varphi\left([\lambda]_{P} \varphi \leftrightarrow \exists c \operatorname{Proof}_{T \mid X}^{\Lambda}(c, \lambda, \varphi, P)\right) .
$$

Then, $[\lambda \mid X]_{T}^{\Lambda} \varphi$ is the $\Pi_{1}^{1}$ formula $\forall P\left(\operatorname{IPC}_{T \mid X}^{\Lambda}(P) \rightarrow[\lambda]_{P} \varphi\right)$, and $\langle\lambda \mid X\rangle_{T}^{\Lambda} \varphi$ is defined as $\neg[\lambda \mid X]_{T}^{\Lambda} \neg \varphi$.

Note that the formulas $[\lambda]_{P} \varphi$ and $\langle\lambda\rangle_{P} \varphi$ are independent of $T, \Lambda$, and $X$ and are merely of complexity $\Delta_{0}^{0}(P)$. Note also that $\operatorname{IPC}_{T \mid X}^{\Lambda}(P)$ is a $\Pi_{3}^{0}(\Lambda, X, P)$ formula since $T$ is elementary presented.

We would like to stress that our notion of oracle provability is rather weak in a sense: a formula is provable if it is a member of every iterated provability class corresponding to that oracle, but it can be the case that there simply are no such iterated provability classes. Consequently, and in the same sense, consistency statements are rather strong: from oracle consistency we may conclude the existence of an iterated provability class corresponding to that oracle. Let us state this explicitly in a lemma whose proof merely follows from the definition. 
Lemma 4.2. $\mathrm{ECA}_{0} \vdash \forall X\left(\langle\lambda \mid X\rangle_{T}^{\Lambda} \top \rightarrow \exists Y \operatorname{IPC}_{T \mid X}^{\Lambda}(Y)\right)$.

However, if we are mainly interested in consistency strength, it is not such a bad thing to work under the assumption that iterated provability classes exist for all oracles.

LEMMA 4.3. Any theory $T$ extending $\mathrm{ECA}_{0}$ is provably equiconsistent with $T+\forall X \exists Y \operatorname{IPC}_{T \mid X}^{\Lambda}(Y)$ over EA. That is,

$$
\mathrm{EA} \vdash T \equiv_{\Pi_{1}^{0}} T+\forall X \exists Y \operatorname{IPC}_{T \mid X}^{\Lambda}(Y) .
$$

Proof. As in [16] we remark that $T+\square_{T} \perp \vdash T+\forall X \exists Y \operatorname{IPC}_{T \mid X}^{\Lambda}(Y) \vdash T$ and that $T+\square_{T} \perp \equiv_{\Pi_{1}^{0}} T$. To see the latter, suppose $T \vdash \square_{T} \perp \rightarrow \pi$ for some $\pi \in \Pi_{1}^{0}$. Reason in $T$ and assume $\square_{T} \pi$ and $\neg \pi$. By provable $\Sigma_{1}^{0}$-completeness, $\square_{T} \neg \pi$. Hence $\square_{T} \perp$ and so $\pi$. Thus, $T \vdash \square_{T} \pi \rightarrow \pi$ whence by Löb's rule, $T \vdash \pi$, and all this argument may be formalized in EA.

The assumption that an IPC exists will sometimes be needed to prove apparently trivial properties of iterated provability; for example, that 0 -provability corresponds to ordinary provability. The proof of the following is straightforward and we omit it.

Lemma 4.4. Fix a theory $T$. It is provable in $\mathrm{ECA}_{0}$ that, if $\Lambda$ has a minimum element $0_{\Lambda}, \varphi$ is any formula and $X$ is any set, then:

1. $\square_{T \mid X} \phi$ implies that $\left[0_{\Lambda} \mid X\right]_{T}^{\Lambda} \phi$, and

2. if $P$ satisfies $\operatorname{IPC}_{T \mid X}^{\Lambda}(P)$, then $\left[0_{\Lambda}\right]_{P} \phi$ implies that $\square_{T \mid X} \phi$.

Fortunately, we can prove that IPCs exist and are unique, but such a proof requires a relatively strong theory. Below, $\exists$ ! is a standard abbreviation for "there exists a unique", and $\exists_{\leq 1}$ for "there exists at most one".

LEMma 4.5. Let $T$ be any elementarily represented theory. Then,

1. $\mathrm{ACA}_{0}$ proves that if $\Lambda$ is a well-order then $\exists_{\leq 1} P \operatorname{IPC}_{T \mid X}^{\Lambda}(P)$, and

2. $\mathrm{ATR}_{0}$ proves that if $\Lambda$ is a well-order then $\exists ! P \operatorname{IPC}_{T \mid X}^{\Lambda}(P)$.

Proof. The first claim is analogous to a result of [16]. Via an easy transfinite induction we see in $\mathrm{ACA}_{0}$ that iterated provability classes are unique, given a wellorder $\Lambda$, an oracle set $X$, and a c.e. base theory $T$. The second is immediate from the first and the fact that $\forall \Lambda\left(\right.$ wo $\left.(\Lambda) \rightarrow \forall X \exists P \operatorname{IPC}_{T \mid X}^{\Lambda}(P)\right)$ is an instance of TR- $\Pi_{\omega}^{0}$.

4.2. Normality and completeness for oracle provability. Many results established in [16] for $[\lambda]_{T}^{\Lambda} \varphi$ simply carry over to $[\lambda \mid X]_{T}^{\Lambda} \varphi$. For example, as an almost immediate consequence of the definition, we get monotonicity on $\lambda$. Moreover, there is also a form of oracle-monotonicity which requires stronger assumptions. The next lemma is a generalization of a result in [16] to theories with oracles.

Lemma 4.6. Let $T$ be any theory.

1. It is provable in $\mathrm{ECA}_{0}$ that, if $\Lambda$ is any linear order, then for every formula $\varphi$, every $\eta<_{\Lambda} \lambda$ and every set $X,[\eta \mid X]_{T}^{\Lambda} \varphi$ implies that $[\lambda \mid X]_{T}^{\Lambda} \varphi$.

2. It is provable in $\mathrm{ACA}_{0}$ that, if $\Lambda$ is a well-order and $X, Y$ are sets such that $\exists P \operatorname{IPC}_{T \mid X}^{\Lambda}(P)$ holds, then for every formula $\varphi$ and every $\lambda<\Lambda,[\lambda \mid X]_{T}^{\Lambda} \varphi$ implies that $[\lambda \mid X, Y]_{T}^{\Lambda} \varphi$. 
Proof. The first claim follows from the definition of an IPC using the transitivity of $<_{\Lambda}$. For the second, it should be noted that a small technical translation between the ' $\varphi$ ' in $[\lambda \mid X, Y]_{T}^{\Lambda} \varphi$ and the ' $\varphi$ ' in $[\lambda \mid X]_{T}^{\Lambda} \varphi$ is needed according to the notational conventions established in Section 2.2. Now using the assumption we fix an IPC $P$ for $T \mid X$, and consider an arbitrary IPC $Q$ for $T \mid X, Y$. Then, we prove by transfinite induction on $\lambda<\Lambda$ that $\forall \varphi\left([\lambda]_{P} \varphi \rightarrow[\lambda]_{Q} \varphi\right)$ holds; since the latter is arithmetical, this induction may be performed within $\mathrm{ACA}_{0}$.

We also see that we have distributivity for our provability predicates $[\lambda \mid X]_{T}^{\Lambda}$.

Lemma 4.7. $\mathrm{ACA}_{0}$ proves that, given a theory $T$, a well-order $\Lambda, \lambda<\Lambda$ and formulas $\varphi_{1}, \varphi_{2},[\lambda \mid X]_{T}^{\Lambda}\left(\varphi_{1} \rightarrow \varphi_{2}\right) \rightarrow\left([\lambda \mid X]_{T}^{\Lambda} \varphi_{1} \rightarrow[\lambda \mid X]_{T}^{\Lambda} \varphi_{2}\right)$.

Proof. See [16, Lemma 6.1]. Note that the addition of an oracle does not affect the proof.

COROLLARY 4.8. It is provable in $\mathrm{ACA}_{0}$ that the provability predicates $[\lambda \mid X]_{T}^{\Lambda}$ satisfy the modal logic $\mathrm{K}$ for each well-order $\Lambda$ and each $\lambda<\Lambda$.

We also can prove various completeness results for our provability predicates. Recall that $\boldsymbol{n}$ denotes the set $\{m: m<n\}$ and $\boldsymbol{\omega}$ the natural numbers, both with their usual ordering.

Lemma 4.9. If $\varphi(X, x) \in \Sigma_{2 m+1}^{0}(X)$ and $m<n \leq \omega$, then

$$
\mathrm{ECA}_{0} \vdash \forall X \forall x\left(\varphi(X, x) \rightarrow[\bar{m} \mid X]_{T}^{n} \varphi(\bar{X}, \dot{x})\right) .
$$

Proof. We proceed by an external induction on $m$ and the subformulas of $\varphi$ by their build. Thus, we assume (3) for each subformula of $\varphi$. Without loss of generality we may assume that negations occur only on atomic formulas, that $\varphi$ is in prenex normal form and that it does not contain subformulas of the form $\forall x \forall y \theta$ or $\exists x \exists y \theta$ where the occurrence of $y$ is unbounded. For the remainder of the proof we reason in $\mathrm{ECA}_{0}$.

For the base case, $\varphi$ is an atomic formula or negation of an atomic formula, which is of one of the following forms: either it contains no second-order variables, in which case we obtain $\square_{T} \varphi(\dot{x})$ by provable $\Sigma_{1}^{0}$-completeness, Lemma 3.2. Otherwise, it is of the form $t \in X$ or $t \notin X$ for some term $t$, which is provably equivalent to an axiom of $T \mid X$.

If $\varphi$ is bounded but not atomic, we merely follow a routine induction on the build of $\varphi$. The case where $\varphi$ is a Boolean combination of its subformulas is straightforward, and bounded quantifiers may be replaced by conjunctions or disjunctions using Lemma 3.3.

If $\varphi=\exists x \theta(x)$, then for some $k$ we have that $\theta(k)$ is true. By the induction hypothesis, $[\bar{m} \mid X]_{T}^{n} \theta(\bar{k})$, and by existential introduction, $[\bar{m} \mid X]_{T}^{n} \exists x \theta(x)$. Finally, we consider the case $\varphi=\forall x \theta$. Since by assumption $\forall x \theta \in \Sigma_{2 m+1}^{0}$, we have that $\forall x \theta \in \Pi_{2 m}^{0}$ since it starts with a universal quantifier, so that $\theta \in \Sigma_{2(m-1)+1}^{0}$. Thus, by the induction hypothesis we have for every $k$ that $[\overline{m-1} \mid X]_{T}^{n} \theta(\dot{k})$ and therefore $[\bar{m} \mid X]_{T}^{n} \forall x \theta(x)$ by one application of the $\omega$-rule.

As an immediate corollary we get $\boldsymbol{\Pi}_{2 m}^{0}$-completeness as well. We also get $\Sigma_{1}^{1}$-completeness for provability involving at least $\omega$-many iterations as is manifest in the next theorem, provided we are allowed to pick a suitable oracle. We will 
write $\left[\lambda \mid X_{0}, \ldots, X_{n}\right]_{T}^{\Lambda}$ for oracle provability where the tuple $X_{0}, \ldots, X_{n}$ is represented by a single set using the conventions from Section 3.

THEOREM 4.10. If $\varphi(X, x) \in \Sigma_{1}^{1}(X)$ then there exists $n$ such that

$$
\mathrm{ECA}_{0} \vdash \forall x \forall X \exists Y\left(\varphi(X, x) \rightarrow[\bar{n} \mid Y, X]_{T}^{\omega} \varphi(\bar{X}, \dot{x})\right) .
$$

Proof. By assumption, $\varphi(X, x)$ is of the form $\exists Y \varphi_{0}(Y, X, x)$ with the formula $\varphi_{0}(Y, X, x) \in \Pi_{n}^{0}(X, Y)$ for some $n$. We now reason in $\mathrm{ECA}_{0}$, fix some $X$ and $x$ and assume $\varphi(X, x)$. Thus, for some $Y$ we have $\varphi_{0}(Y, X, x)$. By Lemma 4.9, we see that $[\bar{n} \mid Y, X]_{T}^{\omega} \varphi_{0}(\bar{Y}, \bar{X}, \dot{x})$, whence also $[\bar{n} \mid Y, X]_{T}^{\omega} \varphi(\bar{X}, \dot{x})$.

5. Oracle consistency and oracle reflection. In this section we will introduce the notions of reflection and consistency that naturally correspond to oracle provability, and relate them both to each other and to arithmetical comprehension.

5.1. Basic definitions. First, let us define the notions of reflection and consistency we are interested in.

Definition 5.1 (Oracle reflection and oracle consistency). For $\Gamma$ a class of formulas not containing any occurrence of $\mathcal{O}$, we define $\lambda$-OracleRFN $\mathrm{Or}_{\Gamma}^{\Lambda}(T)$ (oracle reflection) as the schema

$$
\forall X_{0} \cdots \forall X_{n} \forall x\left(\left[\lambda \mid X_{0}, \ldots, X_{n}\right]_{T}^{\Lambda} \varphi\left(\overline{X_{0}}, \ldots, \overline{X_{n}}, \dot{x}\right) \rightarrow \varphi\left(X_{0}, \ldots, X_{n}, x\right)\right)
$$

for $\varphi\left(X_{0}, \ldots, X_{n}, x\right) \in \Gamma$ and $\operatorname{FV}\left(\varphi\left(X_{0}, \ldots, X_{n}, x\right)\right) \subseteq\left\{X_{0}, \ldots, X_{n}, x\right\}$. We also define

$$
\begin{aligned}
& \lambda \text {-OracleCons }{ }^{\Lambda}(T):=\lambda \text {-OracleRFN }{ }_{\{\perp\}}^{\Lambda}(T) ; \\
& \Lambda \text {-OracleCons }(T):=\forall \lambda<\Lambda\left(\lambda \text {-OracleCons }{ }^{\Lambda}(T)\right) \text {; } \\
& \Lambda \text {-OracleRFN }{ }_{\Gamma}(T):=\forall \lambda<\Lambda\left(\lambda-\operatorname{OracleRFN}_{\Gamma}^{\Lambda}(T)\right) .
\end{aligned}
$$

The next easy example makes clear why we imposed the restriction of $\mathcal{O}$ not occurring in $\Gamma$. Let $X:=\{2\}$ and let $Y:=\varnothing$. Clearly we have $[\lambda \mid X]_{T}^{\Lambda} \mathcal{O}(\overline{2})$ and $[\lambda \mid Y]_{T}^{\Lambda} \neg \mathcal{O}(\overline{2})$ so that we arise at a contradiction where we allowed to apply oracle reflection to these sentences. We shall later see that oracle reflection with the restriction is consistent.

5.2. Oracle reflection and comprehension. Let us see that with just a little amount of oracle reflection we get arithmetical comprehension. Observe that below we do not assume that $\Lambda$ is a well-order, only that it has a minimum element $0_{\Lambda}$.

Lemma 5.2. $\mathrm{ACA}_{0} \subseteq \mathrm{ECA}_{0}+\forall \lambda<\Lambda\left(0_{\Lambda} \leq_{\Lambda} \lambda\right)+0_{\Lambda}$-OracleRFN $\Sigma_{1}^{\Lambda}\left(\mathrm{ECA}_{0}\right)$.

Proof. Without loss of generality, we may restrict our attention to formulas with at most one set parameter, so we fix a $\Sigma_{1}^{0}(X)$ formula $\varphi(x, X)$. Thus, we reason in $\mathrm{ECA}_{0}$ and fix some set $X$. Since reflection implies consistency we know that there is some iterated provability predicate for $X$ (Lemma 4.2). That is, there is some $P$ with $\operatorname{IPC}_{\mathrm{ECA}_{0} \mid X}^{\Lambda}(P)$.

By $\boldsymbol{\Delta}_{0}^{0}$-CA, we can form the set $Z=\left\{z:\left[0_{\Lambda}\right]_{P} \varphi(\bar{z}, \bar{X})\right\}$. We claim that $z \in Z \leftrightarrow$ $\varphi(z, X)$, which finishes the proof, for $\boldsymbol{\Sigma}_{1}^{0}$-CA and $\boldsymbol{\Pi}_{\omega}^{0}$-CA are equivalent over $\mathrm{ECA}_{0}$ (see, e.g., [27, Lemma III.1.3]). 
If $z \in Z$, by Lemma 4.4.2 we have that $\square_{\mathrm{ECA}_{0} \mid X} \varphi(\bar{z}, \bar{X})$, and thus by Lemma 4.4.1, $\left[0_{\Lambda} \mid X\right]_{\mathrm{ECA}_{0}}^{\Lambda} \varphi(\bar{z}, \bar{X})$. By reflection, we obtain $\varphi(z, X)$. On the other hand, if $\varphi(z, X)$, we get by completeness (Lemma 4.9) that $\left[0_{\Lambda} \mid X\right]_{\mathrm{ECA}_{0}}^{\Lambda} \varphi(\bar{z}, \bar{X})$, so that in particular $\left[0_{\Lambda}\right]_{P} \varphi(\bar{z}, \bar{X})$, and thus $z \in Z$.

The upshot of this lemma is that we can perform many of our arguments in $\mathrm{ACA}_{0}$ once we have just a little bit of reflection, as we will do in the next subsection.

5.3. Oracle consistency versus oracle reflection. It will be convenient to observe that consistency and reflection are easily related to each other.

Lemma 5.3. Fix $m<n \leq \omega$. Over $\mathrm{ECA}_{0}$ we have that:

1. $\bar{m}$-OracleRFN $\Pi_{2 m+1}^{n}(T) \equiv \bar{m}$-OracleCons ${ }^{n}(T)$;

2. if $\operatorname{Lim}(\Lambda)$ then $\Lambda$-OracleRFN $\Pi_{1}^{1}(T) \equiv \Lambda$-OracleCons $(T)$.

Proof. For the first claim, we reason in $\mathrm{ECA}_{0}$. It should be clear that $\bar{m}$-OracleRFN $\boldsymbol{\Pi}_{2 m+1}^{n}(T)$ proves $\bar{m}$-OracleCons ${ }^{n}(T)$. For the other direction, fix a formula $\varphi(x) \in \Pi_{2 m+1}^{0}(X)$, and assume that $[\bar{m} \mid X]_{T}^{n} \varphi(\dot{a})$ holds for some $a$. Towards a contradiction, assume that $\neg \varphi(a)$ also holds, so that by Lemma 4.9, we have that $[\bar{m} \mid X]_{T}^{n} \neg \varphi(\dot{a})$. We may then use Lemma 4.7 to do a bit of propositional reasoning and obtain $[\bar{m} \mid X]_{T}^{n} \perp$, contradicting $\bar{m}$-OracleCons ${ }^{n}(T)$.

For the second claim, we once again focus on the right-to-left direction, since the other is immediate. Reason in $\mathrm{ECA}_{0}$ and assume $\Lambda$-OracleCons $(T)$. Let $1_{\Lambda}$ denote the second element in the well-order $\Lambda$. Reasoning as in the first item with $m=1$, we obtain $1_{\Lambda}-\operatorname{OracleRFN} \Lambda_{\Pi_{3}^{0}}^{\Lambda}(T)$, which in view of Lemma 4.6.1 implies $0_{\Lambda}$-OracleRFN $\Sigma_{\Sigma_{1}^{0}}^{\Lambda}(T)$ and we may use Lemma 5.2 to obtain $\mathrm{ACA}_{0}$.

Thus we may continue reasoning in $\mathrm{ACA}_{0}+\Lambda$-OracleCons $(T)$. Fix a formula $\varphi(x) \in \Pi_{1}^{1}(X)$, and assume that $[\lambda \mid X]_{T}^{\Lambda} \varphi(\dot{a})$ for some $\lambda<\Lambda$ and $a$. As before, assume that $\neg \varphi(a)$ holds; then, by Theorem 4.10 there are $m$ and $Y$ such that $\left[m_{\Lambda} \mid X, Y\right]_{T}^{\Lambda} \neg \varphi(\dot{a})$, where $m_{\Lambda}$ denotes the $m$-th element in the well-order $\Lambda$. Letting $\eta$ be the greatest of $\lambda, m_{\Lambda}$ in $\Lambda$, we see using $[\lambda \mid X]_{T}^{\Lambda} \varphi(\dot{a})$ that $[\eta \mid X, Y]_{T}^{\Lambda} \neg \varphi(\dot{a})$ and $[\eta \mid X]_{T}^{\Lambda} \varphi(\dot{a})$ both hold.

Now, using Lemma 4.2, there exists $P$ such that $\operatorname{IPC}_{T \mid X}^{\Lambda}(P)$. Thus we may use $[\eta \mid X]_{T}^{\Lambda} \varphi(\dot{a})$ and Lemma 4.6.2 to obtain $[\eta \mid X, Y]_{T}^{\Lambda} \varphi(\dot{a})$; but since we also had that $[\eta \mid X, Y]_{T}^{\Lambda} \neg \varphi(\dot{a})$, this contradicts $\Lambda$-OracleCons $(T)$.

6. Predicative oracle reflection and consistency. Our notions of oracle reflection and oracle consistency include a particular well-order $\Lambda$. We shall now define what we call predicative oracle reflection and predicative oracle consistency which stipulate the oracle notions for any well-order.

DEFINITION 6.1. We define the principle predicative oracle reflection as the scheme

$$
\text { Pred-0-RFN }{ }_{\Gamma}(T)=\forall \Lambda \forall \lambda<\Lambda\left(\text { wo }(\Lambda) \rightarrow \lambda \text {-0racleRFN }{ }_{\Gamma}^{\Lambda}(T)\right),
$$

and predicative oracle consistency as the statement

$$
\operatorname{Pred-0-Cons}(T)=\forall \Lambda \forall \lambda<\Lambda\left(\text { wo }(\Lambda) \rightarrow \lambda \text {-OracleCons }{ }^{\Lambda}(T)\right) .
$$

Let us first make a simple observation.

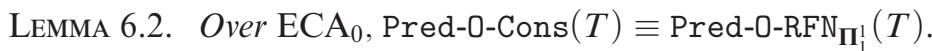

Proof. This follows from Lemma 5.3. 
The main result of this section is that predicative reductionism follows from predicative oracle consistency over a rather weak theory. To be more precise, we shall prove that $\mathrm{ATR}_{0}$ follows from $\mathrm{ECA}_{0}+\operatorname{Pred-0}-\operatorname{Cons}\left(\mathrm{ECA}_{0}\right)$.

6.1. Predicative oracle consistency proves predicative reductionism. Let us recall from Section 3.2 the formula $\operatorname{TR}^{\Lambda}(\varphi, X)$ which says that the set $X$ satisfies transfinite recursion for $\varphi$ over the well-order $\Lambda$. If $\varphi$ is arithmetical, then so is $\operatorname{TR}^{\Lambda}(\varphi, X)$. Moreover, we note that $\operatorname{TR}^{\Lambda}(\varphi, X)$ only imposes restrictions on numbers which code pairs $\langle\xi, n\rangle$ with $\xi \in|\Lambda|$ and says nothing about numbers not of this form.

In order to prove properties of transfinite recursion it will be useful to have restricted versions of $\operatorname{TR}^{\Lambda}(\varphi, X)$. Thus, we define

$$
\operatorname{TR}_{\lambda}^{\Lambda}(\varphi, X):=\forall \xi \leq_{\Lambda} \lambda \forall x\left(x \in X_{\xi} \leftrightarrow \varphi\left(x, X_{<_{\Lambda} \xi}\right)\right),
$$

and $\operatorname{TR}_{<\lambda}^{\Lambda}(\varphi, X):=\forall \xi<{ }_{\Lambda} \lambda \operatorname{TR}_{\xi}^{\Lambda}(\varphi, X)$. The formula $\varphi$ may have many free number and set variables. However, one set variable of $\varphi$ plays a special role in $\operatorname{TR}^{\Lambda}(\varphi, X)$, like the variable $X$ in $\operatorname{TR}_{\lambda}^{\Lambda}(\varphi, X)$, and this variable shall always be displayed.

Sometimes we may wish to emphasize that $\operatorname{TR}_{\lambda}^{\Lambda}(\varphi, Y)$ has other free variables appearing in $\varphi$. We will do so by using a semicolon, e.g., $\operatorname{TR}_{\lambda}^{\Lambda}(\varphi, Y ; X)$. We stipulate that any first-order variable, say $z$, in $\operatorname{TR}_{\lambda}^{\Lambda}(\varphi, Y ; z)$ will be dotted when occurring in the scope of a box. Thus if, for example $z$ is free in $\varphi$, then $\operatorname{TR}_{\lambda}^{\Lambda}(\varphi, X)$ means $\operatorname{TR}_{\lambda}^{\Lambda}(\varphi, X ; z)$, and by definition $[\xi \mid \Lambda]_{T}^{\Lambda} \operatorname{TR}_{\dot{\lambda}}^{\bar{\Lambda}}(\varphi, Y)$ denotes $[\xi \mid \Lambda]_{T}^{\Lambda} \operatorname{TR}_{\dot{\lambda}}^{\bar{\Lambda}}(\varphi, Y ; \dot{z})$.

In order to prove that predicative oracle consistency proves transfinite recursion, given arithmetical $\varphi(z, Z)$ and a well-order $\Lambda$, we need to construct a set $A$ satisfying $\operatorname{TR}^{\Lambda}(\varphi, A)$. We cannot do this directly without assuming $\operatorname{ATR}_{0}$, so instead we would want to consider the set $A$ of all $\langle\lambda, n\rangle$ such that it is provable that $\langle\lambda, n\rangle \in A$. But of course we cannot refer directly to $A$, so instead we will prove that $n$ belongs to any set that is defined by transfinite recursion of $\varphi$ over $\Lambda$; that is, $\forall Z\left(\mathrm{TR}^{\Lambda}(\varphi, Z) \rightarrow n \in Z\right)$. To be precise, we will use a set of the form

$$
\left\{\langle\lambda, n\rangle:\left[f_{\varphi}(\lambda) \mid A\right]_{\mathrm{ECA}_{0}}^{\Lambda} \forall Z\left(\operatorname{TR}_{<\bar{\lambda}}^{\bar{\Lambda}}(\varphi, Z) \rightarrow \varphi\left(\bar{n}, Z_{<\bar{\lambda}}\right)\right)\right\},
$$

for an appropriate function $f_{\varphi}$ and an appropriately chosen set $A$. The exact values will be provided by the following lemma, where we use the function $n \cdot \alpha+m$ described in Lemma 3.4:

LEMma 6.3. For any pair of formulas $\varphi \in \Pi_{2 m}^{0}(X, Z, \Lambda)$ and $\psi \in \Sigma_{2 \ell+1}^{0}(X, Z, \Lambda)$ we have that $\mathrm{ACA}_{0}$ proves

$$
\begin{aligned}
\operatorname{Lim}(\Lambda) \rightarrow \forall & Z, X \forall \lambda<\Lambda\left(\operatorname{TR}_{<\lambda}^{\Lambda}(\varphi, Z ; X) \wedge \psi\left(Z_{<\lambda}, X, \Lambda\right)\right. \\
& \left.\rightarrow[\bar{m} \cdot \lambda+\bar{\ell} \mid X, \Lambda]_{T}^{\Lambda} \forall Z\left(\operatorname{TR}_{<\lambda}^{\bar{\Lambda}}(\varphi, Z ; \bar{X}) \rightarrow \psi\left(Z_{<i}, \bar{X}, \bar{\Lambda}\right)\right)\right) .
\end{aligned}
$$

Proof. We reason in $\mathrm{ACA}_{0}$, assume $\operatorname{Lim}(\Lambda)$, fix $Z$ and $X$ and proceed to prove the claim simultaneously for all subformulas $\theta$ of $\varphi, \neg \varphi$ and of $\psi$. We will assume that $\varphi, \psi$ are in prenex normal form, and that there is no subformula of the form $\forall x \forall y \theta$ or $\exists x \exists y \theta$ where the occurrence of $y$ is unbounded. By using negation normal forms, we may also assume that $\neg$ is only applied to atomic formulas. Similarly, let $\sim \varphi$ be a formula provably equivalent to $\neg \varphi$ also in the above form.

Let $\Theta$ denote the set of all subformulas of $\varphi, \sim \varphi$, and $\psi$. For each $\theta \in \Theta$, we consider the following formula $\gamma_{\theta}(\lambda) \in \Pi_{\omega}^{0}(X, Z, \Lambda)$ : 


$$
\begin{aligned}
& \forall v\left(\operatorname{TR}_{<\lambda}^{\Lambda}(\varphi, Z ; X) \wedge\right. \theta\left(v, Z_{<\lambda}, X, \Lambda\right) \\
&\left.\rightarrow\left[\bar{m} \cdot \lambda+\overline{\ell_{\theta}} \mid X, \Lambda\right]_{T}^{\Lambda} \forall Z\left(\operatorname{TR}_{<\dot{\lambda}}^{\bar{\Lambda}}(\varphi, Z ; \bar{X}) \rightarrow \theta\left(\dot{v}, Z_{<\dot{\lambda}}, \bar{X}, \bar{\Lambda}\right)\right)\right),
\end{aligned}
$$

where $\ell_{\theta}$ is the least natural number such that $\theta \in \Pi_{2 \ell_{\theta}}^{0}(X, Z, \Lambda) \cup \Sigma_{2 \ell_{\theta}+1}^{0}(X, Z, \Lambda)$. Finally, we define $\gamma(\lambda)=\bigwedge_{\theta \in \Theta} \gamma_{\theta}(\lambda) \in \Pi_{\omega}^{0}(X, Z, \Lambda)$.

We reason by transfinite induction in $\mathrm{ACA}_{0}$, assuming that $\forall \mu<\lambda \gamma(\mu)$. In order to derive $\gamma(\lambda)$, we show that $\gamma_{\theta}(\lambda)$ holds for each $\theta \in \Theta$. We prove $\gamma_{\theta}(\lambda)$ by a (secondary) external induction on the build of the subformulas $\theta$ as in the proof of Lemma 4.9. Hence, we have to consider two new base cases.

First we consider the case when $\theta=\theta\left(v, Z_{<\lambda}, X, \Lambda\right)$ is of the form $\left(v_{0}<_{\Lambda} \lambda\right) \wedge(v \in Z)$, in which case we have that $\overline{v_{0}}<\bar{\Lambda} \bar{\lambda}$ is readily derivable from the axioms of $T \mid X, \Lambda$. Meanwhile, by the assumption that $v \in Z$ and $\operatorname{TR}_{<\lambda}^{\Lambda}(\varphi, Z)$, we must have that both $\varphi\left(v_{1}, Z_{<v_{0}}, X, \Lambda\right)$ and $\operatorname{TR}_{<v_{0}}^{\Lambda}(\varphi, Z)$ are true, and by the induction hypothesis applied to $v_{0}$ (notice that $\varphi \in \Theta$ ) we have

$$
\left[\bar{m} \cdot v_{0}+\bar{m} \mid X, \Lambda\right]_{T}^{\Lambda} \forall Z\left(\mathrm{TR}_{<\dot{v}_{0}}^{\bar{\Lambda}}(\varphi, Z ; \bar{X}) \rightarrow \varphi\left(\dot{v}_{1}, Z_{<\dot{v}_{0}}, \bar{X}, \bar{\Lambda}\right)\right) .
$$

Since $\Lambda$ is a limit well-order, in view of Lemma 3.4 we have $m \cdot v_{0}+m \in|\Lambda|$. Since $v_{0}+1 \leq_{\Lambda} \lambda$ it now immediately follows that

$$
[\bar{m} \cdot \lambda \mid X, \Lambda]_{T}^{\Lambda} \forall Z\left(\mathrm{TR}_{<i}^{\bar{\Lambda}}(\varphi, Z ; \bar{X}) \rightarrow\left(\dot{v}_{0}<_{\bar{\Lambda}} \dot{\lambda} \wedge \dot{v} \in Z\right)\right)
$$

as was to be shown. The case where $\theta=\neg\left(\left(v_{0}<_{\Lambda} \lambda\right) \wedge(v \in Z)\right)$ is proven similarly. If $\neg\left(\overline{v_{0}}<\bar{\Lambda} \bar{\lambda}\right)$ holds then this formulas is derivable from the axioms of $T \mid X, \Lambda$. In other case, by the assumption that $\neg(v \in Z)$ and $\operatorname{TR}_{<\lambda}^{\Lambda}(\varphi, Z ; X)$, we have that $\neg \varphi\left(v_{1}, Z_{<v_{0}}, X, \Lambda\right)$ and $\operatorname{TR}_{<v_{0}}^{\Lambda}(\varphi, Z ; X)$ are true, and we conclude as in the previous case (using now that $\sim \varphi \in \Theta$ ).

Other base cases and the induction step for Boolean connectives and unbounded existential quantifiers can be handled as in Lemma 4.9.

Let us show how to deal with bounded quantifiers. Assume that $\theta$ is of the form $\forall u<t(v) \theta_{0}(u, Z)$, and that $\operatorname{TR}_{<\lambda}^{\Lambda}(\varphi, Z ; X)$ and $\theta\left(v, Z_{<\lambda}, X, \Lambda\right)$ hold. Note that $\ell_{\theta}=\ell_{\theta_{0}}=0$; then, by our (external) induction hypothesis, we get

$$
\forall u<t(v)[\bar{m} \cdot \lambda \mid X, \Lambda]_{T}^{\Lambda} \forall Z\left(\mathrm{TR}_{<\dot{\lambda}}^{\bar{\Lambda}}(\varphi, Z ; \bar{X}) \rightarrow \theta_{0}\left(\dot{u}, Z_{<\dot{\lambda}}, \bar{X}, \bar{\Lambda}\right)\right) .
$$

As a consequence,

$$
[\bar{m} \cdot \lambda \mid X, \Lambda]_{T}^{\Lambda} \forall Z\left(\operatorname{TR}_{<\dot{\lambda}}^{\bar{\Lambda}}(\varphi, Z ; \bar{X}) \rightarrow \bigwedge_{u<t(\dot{v})} \theta_{0}\left(\dot{u}, Z_{<\dot{\lambda}}, \bar{X}, \bar{\Lambda}\right)\right) .
$$

In view of Lemma 3.3, it follows that

$$
[\bar{m} \cdot \lambda \mid X, \Lambda]_{T}^{\Lambda} \forall Z\left(\mathrm{TR}_{<i}^{\bar{\Lambda}}(\varphi, Z ; \bar{X}) \rightarrow \theta\left(\dot{v}, Z_{<i}, \bar{X}, \bar{\Lambda}\right)\right) .
$$

Finally, we deal with an unbounded universal quantifier; that is, suppose that $\theta$ is of the form $\forall u \theta_{0}(u, Z, X, \Lambda)$. Then, assuming $\operatorname{TR}_{<\lambda}^{\Lambda}(\varphi, Z)$ and $\theta\left(Z_{<\lambda}, X, \Lambda\right)$, by our (external) induction hypothesis we get

$$
\forall u\left[\bar{m} \cdot \lambda+\overline{\ell_{\theta_{0}}} \mid X, \Lambda\right]_{T}^{\Lambda} \forall Z\left(\mathrm{TR}_{<\dot{\lambda}}^{\bar{\Lambda}}(\varphi, Z ; \bar{X}) \rightarrow \theta_{0}\left(\dot{u}, Z_{<\dot{\lambda}}, \bar{X}, \bar{\Lambda}\right)\right) .
$$

Observe that in this case $\ell_{\theta}=\ell_{\theta_{0}}+1$, and thus by an application of the $\omega$-rule and some reasoning in predicate logic we obtain 


$$
\left[\bar{m} \cdot \lambda+\overline{\ell_{\theta}} \mid X, \Lambda\right]_{T}^{\Lambda} \forall Z\left(\mathrm{TR}_{<\dot{\lambda}}^{\bar{\Lambda}}(\varphi, Z ; \bar{X}) \rightarrow \forall u \theta_{0}\left(u, Z_{<\dot{\lambda}}, \bar{X}, \bar{\Lambda}\right)\right),
$$

as required.

By the above lemma, if $A$ is a set satisfying $\operatorname{TR}^{\Lambda}(\varphi, A ; X)$ and $\langle\lambda, n\rangle \in A$, then we must also have that $[\bar{m} \cdot \lambda+\bar{\ell} \mid X, \Lambda]_{T}^{\Lambda} \forall Z\left(\operatorname{TR}_{<\dot{\lambda}}^{\bar{\Lambda}}(\varphi, Z ; \bar{X}) \rightarrow \dot{n} \in Z_{<\dot{\lambda}}\right)$. If we have reflection for $\Pi_{1}^{1}$ formulas, then setting $Z=A$ we see that the converse implication also holds. Thus we may expect that $A$ satisfies $\widetilde{\mathrm{TR}}_{\lambda}^{\Lambda}(\varphi, A)$, as defined below:

Definition 6.4. Consider $\varphi \in \Pi_{m}^{0}(W, X, \Lambda)$. We define ${\widetilde{\mathrm{TR}_{\lambda}}}_{\lambda}^{\Lambda}(\varphi, A ; W)$ by

$$
\begin{aligned}
& \forall x\left(x_{0} \leq_{\Lambda} \lambda \rightarrow(x \in A\right. \\
& \left.\left.\quad \leftrightarrow\left[\bar{m} \cdot x_{0}+\bar{m} \mid W, \Lambda\right]_{T}^{\Lambda} \forall X\left(\operatorname{TR}_{<\dot{x}_{0}}^{\bar{\Lambda}}(\varphi, X ; \bar{W}) \rightarrow \varphi\left(\dot{x}_{1}, X_{<\dot{x}_{0}}, \bar{W}, \bar{\Lambda}\right)\right)\right)\right) .
\end{aligned}
$$

We also define $\widetilde{\mathrm{TR}}_{<\lambda}^{\Lambda}(\varphi, A)$ by $\forall \eta<_{\Lambda} \lambda \widetilde{\mathrm{TR}}_{\eta}^{\Lambda}(\varphi, A)$, as well as defining $\widetilde{\mathrm{TR}}^{\Lambda}(\varphi, A)$ by $\forall \lambda<\Lambda \widetilde{\mathrm{TR}}_{\lambda}^{\Lambda}(\varphi, A)$.

Roughly, $\widetilde{\mathrm{TR}}_{\lambda}^{\Lambda}(\varphi, A)$ means that the elements of $A$ are precisely those $\langle\eta, n\rangle$ for which it is provable that any set $Z$ satisfying $\operatorname{TR}_{\lambda}^{\Lambda}(\varphi, Z)$ must contain $\langle\eta, n\rangle$. As we will see later, $\widetilde{\operatorname{TR}}_{\lambda}^{\Lambda}(\varphi, A)$ is actually equivalent to $\operatorname{TR}_{\lambda}^{\Lambda}(\varphi, A)$; however, using $\widetilde{\operatorname{TR}}_{\lambda}^{\Lambda}(\varphi, A)$ to define $A$ will be useful to us because the construction of such a set may be carried out using only predicative oracle reflection.

LEMmA 6.5. Given $\varphi \in \Pi_{n}^{0}(W, X, \Lambda)$, we have that

$$
\mathrm{ECA}_{0}+\operatorname{Pred}-0-\operatorname{Cons}\left(\mathrm{ECA}_{0}\right) \vdash \operatorname{Lim}(\Lambda) \rightarrow \forall W \exists X \widetilde{\mathrm{TR}}^{\Lambda}(\varphi, X ; W) .
$$

Proof. Since $\mathrm{ECA}_{0}+$ Pred-0-Cons $\left(\mathrm{ECA}_{0}\right) \vdash \mathrm{ACA}_{0}$, we know that any IPC will be unique given a well-order $\Lambda$ and oracle $W$. So, we reason in $\mathrm{ECA}_{0}+$ Pred-0-Cons $\left(\mathrm{ECA}_{0}\right)$, assume $\operatorname{Lim}(\Lambda)$ and pick $W$ arbitrary as well as $\lambda \in|\Lambda|$. By $[0 \mid W, \Lambda]_{\mathrm{ECA}_{0}}^{\Lambda} \perp \rightarrow \perp$ we observe that $\exists P \operatorname{IPC}_{\mathrm{ECA}_{0} \mid W, \Lambda}^{\Lambda}(P)$. So let $P$ be such an IPC and consider the set

$$
Y:=\left\{\langle\lambda, x\rangle: \lambda \in|\Lambda| \wedge[n \lambda+n]_{P} \forall X\left(\operatorname{TR}_{<\dot{\lambda}}^{\bar{\Lambda}}(\varphi, X ; \bar{W}) \rightarrow \varphi\left(\dot{x}, X_{<i}, \bar{W}, \bar{\Lambda}\right)\right)\right\} .
$$

By $\boldsymbol{\Delta}_{0}^{0}$-comprehension $Y$ is a set and by definition and the uniqueness of an IPC we conclude that $\widetilde{\mathrm{TR}}(\varphi, Y ; W)$.

Lemma 6.6. For $\varphi \in \Pi_{\omega}^{0}(W, X, \Lambda)$ we have that

$$
\mathrm{ECA}_{0}+\operatorname{Pred-0-Cons}\left(\mathrm{ECA}_{0}\right) \vdash \operatorname{Lim}(\Lambda) \rightarrow\left(\widetilde{\mathrm{TR}}^{\Lambda}(\varphi, A) \rightarrow \mathrm{TR}^{\Lambda}(\varphi, A)\right) .
$$

Proof. Fix $\varphi \in \Pi_{2 m}^{0}(W, X, \Lambda)$ and reason in $\mathrm{ECA}_{0}+\operatorname{Pred-0-Cons}\left(\mathrm{ECA}_{0}\right)$. Let $\Lambda, A, W$ be sets such that $\operatorname{Lim}(\Lambda)$, and proceed by transfinite induction on $\lambda<\Lambda$. Note that since all sets are fixed, this induction can be carried out in $\mathrm{ACA}_{0}$, which is available thanks to Lemma 5.2.

So, assume that $\widetilde{\operatorname{TR}}_{\lambda}^{\Lambda}(\varphi, A)$ holds and, by induction, that for all $\eta<\lambda, \operatorname{TR}_{\eta}^{\Lambda}(\varphi, A)$. We need to conclude $\operatorname{TR}_{\lambda}^{\Lambda}(\varphi, A)$ and, since that is equivalent to $\operatorname{TR}_{<\lambda}^{\Lambda}(\varphi, A) \wedge \forall n(n \in$ $\left.A_{\lambda} \leftrightarrow \varphi\left(n, A_{<\lambda}\right)\right)$, it suffices to focus on the second conjunct. 
If $\langle\lambda, n\rangle \in A$, it is immediate by $\Pi_{1}^{1}$ oracle reflection that $\forall X\left(\mathrm{TR}_{<\lambda}^{\Lambda}(\varphi, X) \rightarrow\right.$ $\left.\varphi\left(n, X_{<\lambda}\right)\right)$. But by the induction hypothesis, $\operatorname{TR}_{<\lambda}^{\Lambda}(\varphi, A)$ holds; therefore, in particular, $\varphi\left(n, A_{<\lambda}\right)$ holds as well.

Conversely, if we have that $\varphi\left(n, A_{<\lambda}\right)$, we wish to appeal to Lemma 6.3 to conclude that $\langle\lambda, n\rangle \in A$. By the induction hypothesis, $A$ satisfies $\operatorname{TR}_{<\lambda}^{\Lambda}(\varphi, A)$. Thus from Lemma 6.3,

$$
[m \cdot \lambda+m \mid W, \Lambda]_{T}^{\Lambda} \forall X\left(\mathrm{TR}_{<i}^{\bar{\Lambda}}(\varphi, X ; \bar{W}) \rightarrow \varphi\left(\dot{n}, X_{<i}, \bar{W}, \bar{\Lambda}\right)\right),
$$

and hence $\langle\lambda, n\rangle \in A$. To summarize, we have now shown that for all $x=\langle\eta, n\rangle$ with $\eta \leq \lambda, x \in A \leftrightarrow \varphi\left(n, A_{<\eta}\right)$, that is, $\operatorname{TR}_{\lambda}^{\Lambda}(\varphi, A)$. By transfinite induction on $\lambda$, we conclude that $\operatorname{TR}^{\Lambda}(\varphi, A)$, as claimed.

We can now finally combine all our previous results and formulate the main theorem of this section.

TheOREM 6.7. $\mathrm{ECA}_{0}+\operatorname{Pred}-0-\operatorname{Cons}\left(\mathrm{ECA}_{0}\right) \vdash \mathrm{ATR}_{0}$.

Proof. Immediate from Lemmas 6.5 and 6.6 , bearing in mind that every wellorder $\Lambda$ can be extended to the limit well-order $\Lambda+\omega$.

7. Countable coded $\omega$-models and reflection. Our goal in this section is to derive a converse of Theorem 6.7; in fact, we will even show that $\mathrm{ATR}_{0}$ proves Pred-0-RFN $\Pi_{1}^{1}$ (ACA). The main tool for this task will be the notion of a countable coded $\omega$-model. In what follows we shall discuss existence results for $\omega$-models and the satisfaction definitions associated to them. First we briefly recall the definition and basic properties of these models (we refer to [27, Chapters VII and VIII] for a more detailed account of this topic).

7.1. $\omega$-models and satisfaction definitions. Let $\mathcal{L}_{1}$ denote the usual language of first-order arithmetic augmented with exponentiation. A structure $\mathfrak{M}=\langle\mathfrak{N}, \mathcal{S}\rangle$ for the language of second-order arithmetic $\mathcal{L}_{2}$ is given by an $\mathcal{L}_{1}$-structure $\mathfrak{N}$ together with a family $\mathcal{S}$ of subsets of the universe of $\mathfrak{N}$. An $\omega$-model is just an $\mathcal{L}_{2}$-structure $\mathfrak{M}=\langle\mathfrak{N}, \mathcal{S}\rangle$ where $\mathfrak{N}$ is the standard $\mathcal{L}_{1}$-structure with universe $\omega$. Therefore, in order to fully describe an $\omega$-model it is enough to provide a subset $\mathcal{S}$ of $\mathcal{P}(\omega)$. This motivates the following definition.

Definition 7.1. A countable coded $\omega$-model is a set $\mathfrak{M} \subseteq \mathbb{N}$ viewed as a code for a countable sequence of subsets of $\mathbb{N},\left\{\mathfrak{M}_{n}: n \in \mathbb{N}\right\}$, where for each $n \in \mathbb{N}$, $\mathfrak{M}_{n}=\{i:\langle n, i\rangle \in \mathfrak{M}\}$.

A satisfaction notion can be associated to each countable coded $\omega$-model in a rather natural way. To this end we introduce some auxiliary concepts. We denote by $\mathcal{L}_{C}$ the language obtained by adding to $\mathcal{L}_{2}$ a sequence of new constant symbols $\left\{C_{n}: n \in \mathbb{N}\right\}$. The constants $C_{n}$ are second-order and are used as names for the sets $\mathfrak{M}_{n}$ of the sequence coded by $\mathfrak{M}$.

Let val $: \operatorname{Trm}_{C} \rightarrow \mathbb{N}$ be the standard primitive recursive function that associates to each (Gödel number of a) closed first-order term $t$ of $\mathcal{L}_{C}$ its value val $(t)$ under the usual interpretation for the symbols of $\mathcal{L}_{C}$. Moreover, let $\mathrm{Snt}_{C}$ denote the set of sentences of $\mathcal{L}_{C}$.

DeFINITION 7.2. Let $\mathfrak{M}$ be a countable coded $\omega$-model. A (full) satisfaction definition for $\mathfrak{M}$ is a set $S a t \subseteq \mathrm{Snt}_{C}$ which obeys the usual recursive clauses of Tarski's 
truth definition, where each constant $C_{n}$ is interpreted using $\mathfrak{M}_{n}$. In particular, for every $t, t^{\prime} \in \operatorname{Trm}_{C}$ and $n \in \mathbb{N}$,

$$
\begin{aligned}
& \left(t=t^{\prime}\right) \in S a t \quad \Leftrightarrow \quad \operatorname{val}(t)=\operatorname{val}\left(t^{\prime}\right) ; \\
& \left(t \in C_{n}\right) \in \text { Sat } \Leftrightarrow \operatorname{val}(t) \in \mathfrak{M}_{n} \text {; } \\
& (\neg \varphi) \in \text { Sat } \Leftrightarrow \varphi \notin \text { Sat; } \\
& \left(\varphi_{1} \wedge \varphi_{2}\right) \in \text { Sat } \Leftrightarrow \varphi_{1} \in \text { Sat and } \varphi_{2} \in \text { Sat; } \\
& (\forall u \varphi(u)) \in \text { Sat } \Leftrightarrow \text { for all } n \in \mathbb{N}, \varphi(\bar{n}) \in \text { Sat; } \\
& (\forall X \varphi(X)) \in \text { Sat } \Leftrightarrow \text { for all } n \in \mathbb{N}, \varphi\left(C_{n}\right) \in \text { Sat. }
\end{aligned}
$$

We may assume other connectives and quantifiers are defined in terms of $\neg, \wedge, \forall$. We say that $\mathfrak{M}$ is a full $\omega$-model if there is a full satisfaction definition for $\mathfrak{M}$.

It can be easily shown in $\mathrm{RCA}_{0}$ that if $\mathfrak{M}$ is a countable coded $\omega$-model, then there exists a unique partial satisfaction definition for $\Delta_{0}^{0}$-formulas in $\mathfrak{M}$ (that is, the recursion from Definition 7.2 is only required to hold for $\Delta_{0}^{0}$ sentences of $\mathcal{L}_{C}$ ). Nevertheless, existence of a full satisfaction definition requires a stronger theory, such as $\mathrm{ATR}_{0}$; uniqueness, on the other hand, does not require such a strong base theory.

LEMMA 7.3. RCA 0 proves that for any countable coded $\omega$-model $\mathfrak{M}$, there is at most one full satisfaction definition for $\mathfrak{M}$.

Proof. We shall give a sketch. First we define by primitive recursion a function $d g: \operatorname{Form}\left(\mathcal{L}_{C}\right) \rightarrow \mathbb{N}$, so that $d g(\varphi)$ is the number of quantifiers and connectives occurring in $\varphi$. Then, if $S a t_{1}$ and $S a t_{2}$ are full satisfaction definitions for $\mathfrak{M}$, we can prove by $\Pi_{1}^{0}$-induction on $u$ that

$$
\forall u \forall \varphi \in \operatorname{Snt}_{C}\left(d g(\varphi) \leq u \rightarrow\left(\varphi \in \operatorname{Sat}_{1} \leftrightarrow \varphi \in \operatorname{Sat}_{2}\right)\right) ;
$$

therefore, $S a t_{1}=S a t_{2}$. But $\mathrm{RCA}_{0}$ proves $\boldsymbol{\Pi}_{1}^{0}$-induction (see [27, Corollary II.3.10]), so the result follows.

Definition 7.4. Let $\mathfrak{M}$ be a countable coded $\omega$-model and let $\varphi$ be a sentence of $\mathcal{L}_{C}$. We say that $\mathfrak{M}$ is a full $\omega$-model of $\varphi$ if there is a full satisfaction definition Sat for $\mathfrak{M}$ such that $\varphi \in S a t$, in which case we write $\mathfrak{M}=\varphi$. We say that $\mathfrak{M}$ is a model of a set of formulas $\Phi$ of $\mathcal{L}_{C}$ if, for every $\theta \in \Phi, \mathfrak{M}$ is a model of the universal closure of $\theta$.

Lemma 7.5. Let $\varphi\left(X_{1}, \ldots, X_{m}, v_{1}, \ldots, v_{n}\right) \in \Pi_{\omega}^{0}\left(X_{1}, \ldots, X_{n}\right)$ with all variables shown. It is provable in $\mathrm{ECA}_{0}$ that for every full countable coded $\omega$-model $\mathfrak{M}$ and numbers $a_{1}, \ldots, a_{n}, b_{1}, \ldots, b_{m}$, we have that

$$
\varphi\left(\mathfrak{M}_{a_{1}}, \ldots, \mathfrak{M}_{a_{n}}, b_{1}, \ldots, b_{m}\right) \Longleftrightarrow \mathfrak{M} \models \varphi\left(C_{a_{1}}, \ldots, C_{a_{n}}, \overline{b_{1}}, \ldots, \overline{b_{m}}\right) .
$$

PROOF. Straightforward by (external) induction on the syntactical complexity of the formula $\varphi$, using the Tarskian truth conditions.

LEMMA 7.6. $\mathrm{ECA}_{0}$ proves that for any full countable coded $\omega$-model $\mathfrak{M}$ we have that $\mathfrak{M} \models \mathrm{Q}^{+}+\mathbf{I}_{\omega}^{1}$.

Proof. Reasoning in $\mathrm{ECA}_{0}$, let Sat denote the full satisfaction definition for $\mathfrak{M}$. Since $\mathrm{Q}^{+}$is axiomatized by a true $\Pi_{1}^{0}$-formula, it follows from Lemma 7.5 that $\mathfrak{M} \models \mathrm{Q}^{+}$. Now let $\varphi(u, X) \in \Pi_{\omega}^{1}$ be such that for some $b$

$$
\mathfrak{M} \models \varphi\left(0, \mathfrak{M}_{b}\right) \wedge \forall u\left(\varphi\left(u, \mathfrak{M}_{b}\right) \rightarrow \varphi\left(u+1, \mathfrak{M}_{b}\right)\right) .
$$


Then, $\theta(0, b, \varphi$, Sat $) \wedge \forall x(\theta(x, b, \varphi$, Sat $) \rightarrow \theta(x+1, b, \varphi$, Sat $))$, where $\theta(x, b, \varphi, S a t)$ is the $\Delta_{0}^{0}$-formula $\varphi\left(\bar{x}, C_{b}\right) \in S a t$. By induction we see that $\forall x \theta(x, b, \varphi, S a t)$ and, as a consequence, $\mathfrak{M}=\forall u \varphi\left(u, \mathfrak{M}_{b}\right)$.

7.2. $\omega$-models of $\mathrm{ACA}_{0}$. The following result will be very useful to us; it is a trivial generalization of [27, Theorem VIII.1.13].

Proposition 7.7. Fix a natural number $k$. Then, ATR $\mathrm{A}_{0}$ proves that for any tuple $X_{0}, \ldots, X_{k} \subseteq \mathbb{N}$, there exists a unique, smallest, full countable coded $\omega$-model $\mathfrak{M}$ such that $\mathfrak{M}_{i}=X_{i}$ for all $i \leq k$ and $\mathfrak{M} \models \mathrm{ACA}_{0}$. We will denote this model by $\mathfrak{M}\left[X_{0}, \ldots, X_{k}\right]$.

In view of Lemma 7.6, we immediately obtain the following result (see also [27, Corollary VIII.1.14]):

Corollary 7.8. Proposition 7.7 remains true if we replace $\mathrm{ACA}_{0}$ by $\mathrm{ACA}$ (with full induction). In fact, we already have that $\mathfrak{M}[\vec{X}] \models$ ACA.

Countable coded $\omega$-models can be used for theories with an oracle in which case we only should fix the interpretation of the oracle. For each countable coded $\omega$-model we will adopt the following convention: the oracle $\mathcal{O}$ will always be interpreted using $\mathfrak{M}_{0}$.

Lemma 7.9 (Soundness for $\omega$-models). The following is provable in $\mathrm{ATR}_{0}$. For any $X \subseteq \mathbb{N}$, any well-order $\Lambda$, and any full $\omega$-model $\mathfrak{M}$ for $T \mid X$ with $\mathfrak{M}_{0}=X$, we have for any $\lambda \in|\Lambda|$ and any formula $\varphi$ that if $[\lambda \mid X]_{T}^{\Lambda} \varphi$, then $\mathfrak{M}=\varphi$.

Proof. We reason in $\mathrm{ATR}_{0}$. Given a set $X$, a well-order $\Lambda$ and, a full $\omega$-model $\mathfrak{M}$ for $T \mid X$ with $\mathfrak{M}_{0}=X$, we fix the full satisfaction definition Sat for $\mathfrak{M}$. Using Lemma 4.5.2, let $P$ be the unique set such that $\operatorname{IPC}_{T \mid X}^{\Lambda}(P)$ holds. By transfinite induction we show that $\forall \lambda<\Lambda \forall \varphi\left([\lambda]_{P} \varphi \rightarrow \varphi \in\right.$ Sat $)$.

For $\lambda=0$ this follows from the assumption that $\mathfrak{M} \models T \mid X$, and the inductive step is trivial since we work with $\omega$-models.

7.3. Proving predicative reflection. We are almost ready to state and prove our main theorem. We only need the following lemma.

Lemma 7.10. Let $T$ in $\mathcal{L}_{2}$ be any formal theory such that it is provable in $\mathrm{ATR}_{0}$ that every set $X$ can be included in a full $\omega$-model for $T$. Then,

$$
\mathrm{ATR}_{0} \vdash \operatorname{Pred}-0-\mathrm{RFN}_{\Pi_{2}^{1}}(T) .
$$

Proof. Given a theory $T$ meeting the requirements of the lemma, we fix some $\varphi(X, x) \in \Pi_{2}^{1}(X)$ and reason in $\operatorname{ATR}_{0}$. Let $\Lambda$ be such that wo $(\Lambda)$ and $\lambda \in|\Lambda|$. Now assume that for $A \subseteq \mathbb{N}$ and $a \in \mathbb{N}$ we have $[\lambda \mid A]_{T}^{\Lambda} \varphi(\bar{A}, \dot{a})$. Since $\varphi(X, x) \in \Pi_{2}^{1}(X)$, we can assume that $\varphi(X, x)$ is of the form $\forall Y \exists Z \theta(X, Y, Z, x)$ for some formula $\theta(X, Y, Z, x) \in \Pi_{\omega}^{0}(X, Y, Z)$.

Let $B \subseteq \mathbb{N}$ be arbitrary. We shall show that $\theta(A, B, C, a)$ holds for some set $C$. In view of Lemma 4.6.2 and the fact that $\mathrm{ATR}_{0}$ can prove the existence of IPCs, it follows from $[\lambda \mid A]_{T}^{\Lambda} \varphi(\bar{A}, \dot{a})$, that $[\lambda \mid A, B]_{T}^{\Lambda} \exists Z \theta(\bar{A}, \bar{B}, Z, \dot{a})$. By the hypothesis on $T$, there exists a full $\omega$-model $\mathfrak{M}=\mathfrak{M}[A, B]$ for $T$, so that $\mathfrak{M}_{0}=A$ and $\mathfrak{M}_{1}=B$. By soundness for $\omega$-models (Lemma 7.9), we conclude that $\mathfrak{M} \models \exists Z \theta\left(C_{0}, C_{1}, Z, \bar{a}\right)$, so that for some $b$ we have $\mathfrak{M} \models \theta\left(C_{0}, C_{1}, C_{b}, \bar{a}\right)$. By Lemma 7.5 , for this $b$ we have 
that $\theta\left(A, B, \mathfrak{M}_{b}, a\right)$, and hence $\exists Z \theta(A, B, Z, \bar{a})$ holds. Since $B$ was arbitrary, we conclude that $\varphi$.

We may now summarize our results in the main theorem of this article.

THEOREM 7.11. Let $U, T$ be c.e. theories such that $\mathrm{ECA}_{0} \subseteq U \subseteq \mathrm{ATR}_{0}$, $\mathrm{ECA}_{0} \subseteq T$ and such that $\mathrm{ATR}_{0}$ proves that any set $X$ can be included in a full $\omega$-model for $T$. Then,

$$
\operatorname{ATR}_{0} \equiv U+\operatorname{Pred-0-Cons}(T) \equiv U+\operatorname{Pred-0-RFN} \mathbf{\Pi}_{2}^{1}(T) .
$$

Proof. It is obvious that the third theory is at least as strong as the second, and the other inclusions are Theorem 6.7 and Lemma 7.10.

The following is then immediate in view of Proposition 7.7 and Corollary 7.8:

Corollary 7.12. Let $\mathcal{G}=\left\{\mathrm{ECA}_{0}, \mathrm{RCA}_{0}^{*}, \mathrm{RCA}_{0}, \mathrm{ACA}_{0}\right\}$. Then, (4) holds whenever $U \in \mathcal{G} \cup\left\{\mathrm{ATR}_{0}\right\}$ and $T$ is any theory from $\mathcal{G}$ possibly augmented with full induction.

8. Acknowledgements. All authors were funded via Grant MTM2014-59178-P from the Spanish government. In addition, David Fernández-Duque's work was partially funded by ANR-11-LABX-0040-CIMI within the program ANR-11IDEX-0002-02. Joost J. Joosten received further support from Grant 2014 SGR 437 from the Catalan Government.

Further, we would like to thank Jeremy Avigad, Carl Mummert, Michael Rathjen, Henry Towsner, and Albert Visser for fruitful discussions and/or comments. We are also much indebted to an anonymous referee who pointed out various inaccuracies and who substantially helped to improve the paper.

\section{REFERENCES}

[1] M. BeEson and A. ŠČEDrov, Church's thesis, continuity, and set theory, this Journal, vol. 49 (1984), no. 2, pp. 630-643.

[2] L. D. BEKLEMISHEV, Induction rules, reflection principles, and provably recursive functions. Annals of Pure and Applied Logic, vol. 85 (1997), pp. 193-242.

[3] - Provability algebras and proof-theoretic ordinals, I. Annals of Pure and Applied Logic, vol. 128 (2004), pp. 103-124.

[4] - Veblen hierarchy in the context of provability algebras, Logic, Methodology and Philosophy of Science, Proceedings of the Twelfth International Congress (P. Hájek, L. Valdés-Villanueva, and D. Westerståhl, editors), Kings College Publications, London, 2005, pp. 65-78.

[5] - Reflection principles and provability algebras in formal arithmetic. Russian Mathematical Surveys, vol. 60 (2005), pp. 197-268.

[6] - The Worm principle, Logic Colloquium 2002 (Z. Chatzidakis, P. Koepke, and W. Pohlers, editors), Lecture Notes in Logic 27, ASL Publications, 2006, pp. 75-95.

[7] - On the reduction property for GLP-algebras. Doklady: Mathematics, vol. 472 (2017), no. 4.

[8] L. D. Beklemishev, D. Fernández-Duque, and J. J. Joosten, On provability logics with linearly ordered modalities. Studia Logica, vol. 102 (2014), pp. 541-566.

[9] L. D. BeKlemishev and A. A. OnOprienKo, On some slowly terminating term rewriting systems. Sbornik: Mathematics, vol. 206 (2015), no. 9, pp. 1173-1190.

[10] G. S. Boolos, The Logic of Provability, Cambridge University Press, Cambridge, 1993.

[11] A. Cordón-Franco, A. Fernández-Margarit, and F. F. Lara-Martín, Fragments of Arithmetic and true sentences. Mathematical Logic Quarterly, vol. 51 (2005), pp. 313-328.

[12] S. Feferman, Systems of predicative analysis, this JournaL, vol. 29 (1964), pp. 1-30.

[13] — Systems of predicative analysis II, this JournaL, vol. 33 (1968), pp. 193-220. 
[14] D. FernándeZ-DuQue, The polytopologies of transfinite provability logic. Archive for Mathematical Logic, vol. 53 (2014), no. 3-4, pp. 385-431.

[15] D. Fernández-DuQue and J. J. Joosten, Models of transfinite provability logics, this JournaL, vol. 78 (2013), no. 2, pp. 543-561.

[16] — , The omega-rule interpretation of transfinite provability logic, (2013), arXiv, vol. 1205.2036 [math.LO].

[17] - Well-orders in the transfinite Japaridze algebra. Logic Journal of the Interest Group in Pure and Applied Logic, vol. 22 (2014), no. 6, pp. 933-963.

[18] P. HÁJEK and P. PudLÁK, Metamathematics of First Order Arithmetic, Springer-Verlag, Berlin, Heidelberg, New York, 1993.

[19] J. L. HIRST, A survey of the reverse mathematics of ordinal arithmetic, Reverse Mathematics 2001, Lecture Notes in Logic, vol. 21, A. K. Peters, Natick, MA, 2005, pp. 222-234.

[20] K. N. IGNATIEV, On strong provability predicates and the associated modal logics, this JouRNAL, vol. 58 (1993), pp. 249-290.

[21] G. JAPARIDZE, The polymodal provability logic, Intensional Logics and Logical Structure of Theories: Material from the Fourth Soviet-Finnish Symposium on Logic, Metsniereba, Telaviv, 1988, pp. 16-48, In Russian.

[22] J. J. Joosten, $\Pi_{1}^{0}$-ordinal analysis beyond first-order arithmetic. Mathematical Communications, vol. 18 (2013), pp. 109-121.

[23] G. KREISEL and A. LÉvy, Reflection principles and their use for establishing the complexity of axiomatic systems. Zeitschrift für mathematische Logik und Grundlagen der Mathematik, vol. 14 (1968), pp. $97-142$.

[24] D. LEIVANT, The optimality of induction as an axiomatization of arithmetic, this JouRnAL, vol. 48 (1983), pp. 182-184.

[25] U. R. SCHMERL, A fine structure generated by reflection formulas over primitive recursive arithmetic, Logic Colloquium '78 (Mons, 1978) (M. Boffa, D. Dalen, and K. Mcaloon, editors), Studies in Logic and the Foundations of Mathematics, vol. 97, North-Holland, Amsterdam, 1979, pp. 335-350.

[26] S. G. Simpson, Friedman's research on subsystems of second-order arithmetic, Harvey Friedman's Research in the Foundations of Mathematics (L. Harrington, M. Morley, A Ščedrov, and S. G. Simpson, editors), North-Holland, Amsterdam, 1985, pp. 137-159.

[27] - Subsystems of Second Order Arithmetic, Cambridge University Press, New York, 2009

[28] S. G. Simpson and R. L. Smith, Factorization of polynomials and $\Sigma_{1}^{0}$ induction. Annals of Pure and Applied Logic, vol. 31 (1986), pp. 289-306.

[29] W. TAIT, Finitism. Journal of Philosophy, vol. 78 (1981), pp. 524-546. 